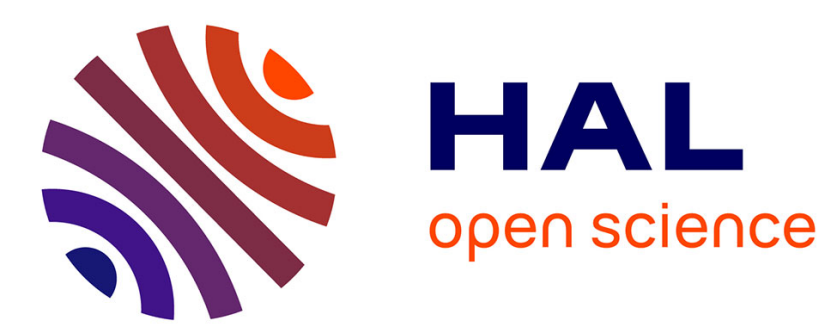

\title{
Nonlocal elasticity tensors in dislocation and disclination cores
}

\author{
V. Taupin, K. Gbemou, Claude Fressengeas, L. Capolungo
}

\section{To cite this version:}

V. Taupin, K. Gbemou, Claude Fressengeas, L. Capolungo. Nonlocal elasticity tensors in dislocation and disclination cores. Journal of the Mechanics and Physics of Solids, 2017, 100, pp.62-84. 10.1016/j.jmps.2017.01.003 . hal-02907618

\section{HAL Id: hal-02907618 \\ https://hal.univ-lorraine.fr/hal-02907618}

Submitted on 27 Jul 2020

HAL is a multi-disciplinary open access archive for the deposit and dissemination of scientific research documents, whether they are published or not. The documents may come from teaching and research institutions in France or abroad, or from public or private research centers.
L'archive ouverte pluridisciplinaire HAL, est destinée au dépôt et à la diffusion de documents scientifiques de niveau recherche, publiés ou non, émanant des établissements d'enseignement et de recherche français ou étrangers, des laboratoires publics ou privés. 


\title{
Nonlocal elasticity tensors in dislocation and disclination cores
}

\author{
${ }^{1 *} \mathrm{~V}$. Taupin, ${ }^{1} \mathrm{~K}$. Gbemou, ${ }^{1} \mathrm{C}$. Fressengeas, ${ }^{2}$ L. Capolungo \\ ${ }^{1}$ Laboratoire d'Etude des Microstructures et de Mécanique des Matériaux, \\ Université de Lorraine/CNRS, Ile du Saulcy, 57045 Metz Cedex, France \\ ${ }^{2}$ Materials Science and Technology Division, Los Alamos National Laboratory, Los \\ Alamos, NM87544, USA
}

\begin{abstract}
Nonlocal elastic constitutive laws are introduced for crystals containing defects such as dislocations and disclinations. In addition to pointwise elastic moduli tensors adequately reflecting the elastic response of defect-free regions by relating stresses to strains and couple-stresses to curvatures, elastic cross-moduli tensors relating strains to couple-stresses and curvatures to stresses within convolution integrals are derived from a nonlocal analysis of strains and curvatures in the defects cores. Sufficient conditions are derived for positive-definiteness of the resulting free energy, and stability of elastic solutions is ensured. The elastic stress/couple stress fields associated with prescribed dislocation/disclination density distributions and solving the momentum and moment of momentum balance equations in periodic media are
\end{abstract}

\footnotetext{
${ }^{*}$ Corresponding author: vincent.taupin@univ-lorraine.fr
} 
determined by using a Fast Fourier Transform spectral method. The convoluted cross-moduli bring the following results: (i) Nonlocal stresses and couple stresses oppose their local counterparts in the defects core regions, playing the role of restoring forces and possibly ensuring spatio-temporal stability of the simulated defects, (ii) The couple stress fields are strongly affected by nonlocality. Such effects favor the stability of the simulated grain boundaries and allow investigating their elastic interactions with extrinsic defects, (iii) Driving forces inducing grain growth or refinement derive from the self-stress and couple stress fields of grain boundaries in nanocrystalline configurations.

\section{Introduction}

In conventional macroscopic modeling of crystalline materials, the elastic constitutive laws relate the elastic strains to their work conjugates, i.e. the stresses. Most of these relationships are local, meaning that they relate the stresses at a given material point of the body to strains at the same point. In such formulations, each individual sub-body is therefore assumed to have its own elastic behavior, independent of the behavior of the neighboring sub-bodies. Local constitutive relationships are valid when the res-

olution length scale, i.e. the characteristic size of the smallest envisionned elementary sub-body, is sufficiently large to ensure that the latter contains a large number of atoms, and possibly of crystal defects. Thus, the constitutive laws characterize the average behavior of some crystalline neighborhood. 
Local elasticity laws may become inappropriate when the resolution length scale is reduced down to or below lattice spacing, particularly if the envisionned sub-bodies involve crystal defects such as dislocations and disclinations. Indeed, due to atomic interactions, the mechanical behavior of a particular atom depends on the relative motion of its neighbors, the latter being strongly disturbed in the presence of crystal defects. Among others, A.C. Eringen and co-workers have devoted substantial work to showing that local constitutive laws cannot render properly the elastic behavior at this scale [Eringen,1966, Eringen,1972, Eringen,1977a, Eringen,1977b, Eringen,2002]. Perhaps even more deeply, defining the stress tensor in a pointwise manner at the scale of a lattice spacing may prove to be an impossible task due to spatial heterogeneity, as implied by the work of [Hardy,1982, Irvin-Kirkwood,1950, Murdoch-Bedeaux,1994, Noll,1955, Tsai,1979] and [Murdoch,2007]. In Eringen's work, nonlocal elastic laws use convolution integrals, where the stresses at a given material point depend on the elastic strains at neighboring points, and where the range of the interactions is controlled by a nonlocal kernel [Eringen,2002]. Such formulations have found several domains of application [Di Paola,2013]. At inter-atomic scale, they allowed retrieving phonon dispersion relations in agreement with lattice dynamics, while the range of nonlocal elasticity was found to be of the order of lattice spacings [Eringen,1977a]. They also allowed regularizing the elastic fields of discrete dislocations, cracks and disclinations [Eringen,1977b, Lazar,2011, Eringen,2002]. The latter result has also been obtained by using couple stresses to describe mechani- 
cal loading, and therefore introducing higher-order constitutive relationships [Lazar,2003, Po,2014, Seif,2014, Lazar,2015]. Since accounting for couple stresses at some material point reflects mechanical forces applied at distant points in the body, such approaches amount to introducing internal length scales in the constitutive formulation, just like convolution integrals.

In this paper, we intend to show that both convolution integrals and higherorder elasticity functionals can be further used to derive nonlocal elastic constitutive laws that achieve full significance in the defected parts of the crystals, where dislocations and disclinations are present. Such effort is made within the context of a recent continuum mechanical framework of crystal defects [Fressengeas,2011], where the discontinuities of the elastic displacement and rotation vector fields induced by the presence of dislocations and disclinations are rendered continuously by the dislocation and disclination density tensor fields [Nye,1953, deWit,1970]. Such a framework is capable of predicting in a fully continuous manner both the core structure and the elastic fields of the defects, in particular those of grain boundaries [Fressengeas,2014, Cordier,2014, Sun,2016]. As a consequence, both the strain and curvature tensors are involved in the constitutive relationships, together with their work conjugates, i.e. the stress and couple stress tensors respectively [Upadhyay,2013]. In the latter reference, it was shown in particular, using linear isotropic elasticity that, in addition to the elastic moduli tensors relating stresses to strains and couple stresses 
to curvatures, the elastic cross-moduli relating stresses to curvatures and couple stresses to strains vanish in the perfectly ordered parts of the crystal because centro-symmetry holds, but become non-zero in the defected parts where centro-symmetry is broken. If only for dimensionality reasons, these cross-moduli are nonlocal in nature, and they reflect elastic behavior that is specific to the defected areas. That the elastic response is different in ordered and disordered regions of the crystals was indeed recently emphasized by analyses of grain boundaries and hetero-phase boundaries [Spearot,2007, Spearot,2008, Tschopp,2008, Dingreville,2014]. Following [Upadhyay,2013] and building on Eringen-type nonlocal elastic energy density functionals for strains and curvatures, we shall derive nonlocal elasticity tensors that exist in defected areas, but vanish in the perfectly ordered regions of the crystals even in anisotropic elasticity. Nonlocality will be shown to derive naturally from kinematic relations existing between the (strongly heterogeneous) strain and curvature fields in the defected areas. A general formulation for these elastic cross-moduli tensors will be proposed, and simplified expressions will be derived to analyze their elasto-static effects on several dislocation and disclination configurations.

The remainder of the paper is organized as follows. In Section 2, useful notations are settled. For completeness, the dislocation and disclination field approach of incompatibility is briefly recalled in Section 3. In Section 4, we develop Eringen-type nonlocal elastic constitutive laws and present a general 
formulation for the cross-moduli relating curvatures to stresses and strains to couple stresses. An admissible simplified formulation of the cross-moduli is proposed in Section 5. Sufficient conditions for positiveness of the free energy density are discussed in Section 6. In Section 7, we present a numerical spectral method using Fast Fourier Transforms to efficiently solve the field equations of the resulting elasto-static boundary value problem in periodic media. Results are presented in Section 8 for several configurations of single dislocations, disclinations and grain boundaries built from disclination dipoles. Conclusions follow.

\section{Notations}

A bold symbol denotes a tensor. When there may be ambiguity, an arrow is superposed to represent a vector: $\overrightarrow{\mathbf{V}}$. The symmetric part of tensor $\mathbf{A}$ is denoted $\mathbf{A}^{\text {sym }}$. Its skew-symmetric and deviatoric parts are $\mathbf{A}^{\text {skew }}$ and $\mathbf{A}^{d e v}$ respectively. The tensor $\mathbf{A} \cdot \mathbf{B}$, with rectangular Cartesian components $A_{i k} B_{k j}$, results from the dot product of tensors $\mathbf{A}$ and $\mathbf{B}$, and $\mathbf{A} \otimes \mathbf{B}$ is their tensorial product, with components $A_{i j} B_{k l}$. The vector $\mathbf{A} \cdot \mathbf{V}$, with rectangular Cartesian components $A_{i j} V_{j}$, results from the dot product of tensor $\mathbf{A}$ and vector $\mathbf{V}$. A : represents the trace inner product of the two second order tensors $\mathbf{A}: \mathbf{B}=A_{i j} B_{i j}$, in rectangular Cartesian components, or the product of a higher order tensor with a second order tensor, e.g., $\mathbf{A}: \mathbf{B}=A_{i j k l} B_{k l}$. The cross product of a second-order tensor $\mathbf{A}$ and a vector $\mathbf{V}$, the div and curl operations for second-order tensors are defined 
row by row, in analogy with the vectorial case. For any base vector $\mathbf{e}_{i}$ of the reference frame:

$$
\begin{aligned}
(\mathbf{A} \times \mathbf{V})^{t} \cdot \mathbf{e}_{i} & =\left(\mathbf{A}^{t} \cdot \mathbf{e}_{i}\right) \times \mathbf{V} \\
(\operatorname{div} \mathbf{A})^{t} \cdot \mathbf{e}_{i} & =\operatorname{div}\left(\mathbf{A}^{t} \cdot \mathbf{e}_{i}\right) \\
(\operatorname{curl} \mathbf{A})^{t} \cdot \mathbf{e}_{i} & =\operatorname{curl}\left(\mathbf{A}^{t} \cdot \mathbf{e}_{i}\right) .
\end{aligned}
$$

In rectangular Cartesian components:

$$
\begin{aligned}
(\mathbf{A} \times \mathbf{V})_{i j} & =e_{j k l} A_{i k} V_{l} \\
(\operatorname{div} \mathbf{A})_{i} & =A_{i j, j} \\
(\operatorname{curlA})_{i j} & =e_{j k l} A_{i l, k} .
\end{aligned}
$$

where $e_{j k l}$ is a component of the third-order alternating Levi-Civita tensor $\mathbf{X}$. A vector $\overrightarrow{\mathbf{A}}$ is associated with tensor $\mathbf{A}$ by using its trace inner product with tensor $\mathbf{X}$ :

$$
(\overrightarrow{\mathbf{A}})_{k}=-\frac{1}{2}(\mathbf{A}: \mathbf{X})_{k}=-\frac{1}{2} e_{i j k} A_{i j}
$$

In the component representation, the spatial derivative with respect to a Cartesian coordinate is indicated by a comma followed by the component index. A superposed dot represents a material time derivative. 


\section{Dislocation and disclination fields, lattice incompatibility}

A small strain setting is assumed. The material displacement field vector $\mathbf{u}$ is single-valued at any point of the crystal and defined continuously. The distortion tensor, defined as the gradient of the displacement $\mathbf{U}=\operatorname{grad} \mathbf{u}$, is curl-free:

$$
\operatorname{curl} \mathrm{U}=0 \text {. }
$$

The strain tensor $\boldsymbol{\epsilon}$ is the symmetric part of $\mathbf{U}$, the rotation tensor $\boldsymbol{\omega}$ its skew-symmetric part and the associated rotation vector $\overrightarrow{\boldsymbol{\omega}}$ is:

$$
\overrightarrow{\boldsymbol{\omega}}=-\frac{1}{2} \boldsymbol{\omega}: \mathbf{X}=\frac{1}{2} \operatorname{curl} \mathbf{u}
$$

Assuming that the displacement vector field and, consequently, the rotation vector field are defined continuously and uniquely at any point of the crystal, including between atoms, amounts to assuming in a dual way that matter is able to transmit stresses and couple stresses at this scale, i.e. below inter-atomic length scale. Such continuity assumptions were made as early as [Schrödinger,1927, Feynman,1939] and [Pauli,1951] in defining pressure and stresses in quantum systems, and later on discussed more extensively by [Nielsen-Martin,1985] in defining the stress tensor in a quantum-mechanical theory of stresses. As already noticed, accounting for couple stresses at some point reflects mechanical forces applied at distant points in the body, and amounts to introducing nonlocality in the description of the mechanical state 
of the body.

Dislocations are introduced by using the dislocation density second order tensor $\boldsymbol{\alpha}$, partially introduced by [Nye,1953]. This areal density tensor has components $\alpha_{i j}=B_{i} t_{j}$ in the reference cartesian frame $\left(\mathbf{e}_{\mathbf{1}}, \mathbf{e}_{\mathbf{2}}, \mathbf{e}_{\mathbf{3}}\right)$. The surface dislocation density is $B_{i}=n b / \Delta S \mathbf{e}_{\mathbf{i}}$, where $n$ is the amount of dislocations (not necessarily an integer) of Burgers vector $\mathbf{b}$ along direction $\mathbf{e}_{\mathbf{i}}$ and of magnitude $b . \Delta S$ denotes the resolution surface. The components of the Nye tensor are thus bearing a Burgers vector length along direction $\mathbf{e}_{i}$ per unit resolution surface $\Delta S, B_{i}$, for a dislocation line vector component along direction $\mathbf{e}_{j}, t_{j}$. In this work, the resolution length scale will very small, i.e., below the Burgers vector magnitude. Hence, a single given dislocation will be described by an appropriate spatial distribution of the dislocation density over the dislocation core area, each local value involving an elementary differential part of the total Burgers vector of the dislocation. In contrast, if the resolution length scale is mesoscopic, the local value of the dislocation density rather reflects the net Burgers vector of a polarized ensemble of dislocation lines. Due to the elastic/plastic displacement discontinuity associated with a polar dislocation density, the plastic $\mathbf{U}_{\mathbf{p}}$ and elastic $\mathbf{U}_{\mathbf{e}}$ components of the total distortion contain incompatible parts $\mathbf{U}_{\mathbf{p}}^{\perp}$ and $\mathbf{U}_{\mathbf{e}}^{\perp}$, which are not curl-free, in addition to the usual curl-free compatible parts, $\mathbf{U}_{\mathbf{e}}^{\|}$and $\mathbf{U}_{\mathbf{p}}^{\|}$. 
The following relations then hold [Acharya,2001]:

$$
\begin{aligned}
\mathbf{U} & =\mathbf{U}_{\mathbf{e}}+\mathbf{U}_{\mathbf{p}} \\
\mathbf{U}_{\mathbf{e}} & =\mathbf{U}_{\mathbf{e}}^{\perp}+\mathbf{U}_{\mathbf{e}}^{\|} \\
\mathbf{U}_{\mathbf{p}} & =\mathbf{U}_{\mathbf{p}}^{\perp}+\mathbf{U}_{\mathbf{p}}^{\|} \\
0 & =\mathbf{U}_{\mathbf{e}}^{\perp}+\mathbf{U}_{\mathbf{p}}^{\perp} \\
\operatorname{curl} \mathbf{U}_{\mathbf{e}}^{\perp} & =-\operatorname{curl} \mathbf{U}_{\mathbf{p}}^{\perp}=\boldsymbol{\alpha} \neq 0 .
\end{aligned}
$$

The incompatibility equations $(13,14)$ define the incompatible plastic distortion $\mathbf{U}_{\mathbf{p}}^{\perp}$ associated with the presence of the Nye's dislocation density tensor as well as the incompatible elastic distortion $\mathbf{U}_{\mathbf{e}}^{\perp}$ offsetting the latter and ensuring the continuity of the total displacement implied by Eq.(8). Since $\mathbf{U}_{\mathbf{e}}^{\|}$and $\mathbf{U}_{\mathbf{p}}^{\|}$are curl-free, Eq.(14) can also be written as [Kröner,1980]:

$$
\operatorname{curl} \mathrm{U}_{\mathrm{e}}=-\operatorname{curl} \mathrm{U}_{\mathrm{p}}=\boldsymbol{\alpha} .
$$

As indicated above, the rotation vector field $\vec{\omega}$ is continuous and single-valued in the body. The gradient of the rotation vector is the second order curvature tensor $\kappa$ :

$$
\kappa=\operatorname{grad} \vec{\omega}
$$

The total curvature tensor is thus a compatible curl-free tensor. To introduce disclinations, we use the deWit's disclination density second order tensor $\boldsymbol{\theta}$ 
[deWit,1970]. It is an areal density tensor, just like the dislocation density tensor. It has components $\theta_{i j}=\Omega_{i} t_{j}$ in the reference cartesian frame $\left(\mathbf{e}_{\mathbf{1}}, \mathbf{e}_{\mathbf{2}}, \mathbf{e}_{\mathbf{3}}\right)$. The surface disclination density is $\Omega_{i}=n \Omega / \Delta S \mathbf{e}_{\mathbf{i}}$, where $n$ is the amount of disclinations (not necessarily an integer) of Frank vector $\Omega$ around $\mathbf{e}_{\mathbf{i}}$ and of magnitude $\Omega$. The components of the deWit's tensor are thus bearing a Frank vector angle around direction $\mathbf{e}_{i}$ per unit resolution surface $\Delta S, \Omega_{i}$, for a disclination line vector component along direction $\mathbf{e}_{j}$, $t_{j}$. Again, if the resolution length scale is very small, a single disclination is described by a spatial distribution of the disclination density over the disclination core region, each local value bearing an elementary Frank vector of the total disclination. However, for a mesoscopic resolution length scale, the local value of the disclination density rather reflects the net Frank vector of a polarized ensemble of disclination lines. Due to the elastic/plastic rotation discontinuity associated with a polar disclination density, the plastic $\boldsymbol{\kappa}_{p}$ and elastic $\boldsymbol{\kappa}_{e}$ components of the total curvature contain incompatible parts $\boldsymbol{\kappa}_{p}^{\perp}$ and $\boldsymbol{\kappa}_{e}^{\perp}$, which are not curl-free, in addition to compatible components, $\boldsymbol{\kappa}_{e}^{\|}$ and $\boldsymbol{\kappa}_{p}^{\|}$. This leads to the following equations:

$$
\begin{aligned}
\boldsymbol{\kappa} & =\boldsymbol{\kappa}_{e}+\boldsymbol{\kappa}_{p} \\
\boldsymbol{\kappa}_{e} & =\boldsymbol{\kappa}_{e}^{\perp}+\boldsymbol{\kappa}_{e}^{\|} \\
\boldsymbol{\kappa}_{p} & =\boldsymbol{\kappa}_{p}^{\perp}+\boldsymbol{\kappa}_{p}^{\|} \\
0 & =\boldsymbol{\kappa}_{e}^{\perp}+\boldsymbol{\kappa}_{p}^{\perp} \\
\operatorname{curl} \boldsymbol{\kappa}_{e}^{\perp} & =-\operatorname{curl} \boldsymbol{\kappa}_{p}^{\perp}=\boldsymbol{\theta} \neq 0 .
\end{aligned}
$$


The incompatibility equations $(20,21)$ define the incompatible plastic curvature $\boldsymbol{\kappa}_{p}^{\perp}$ associated with the presence of a disclination density tensor and the incompatible elastic curvature $\boldsymbol{\kappa}_{e}^{\perp}$ offsetting the latter and ensuring the continuity of the total rotation. Because $\boldsymbol{\kappa}_{e}^{\|}$and $\boldsymbol{\kappa}_{p}^{\|}$are curl-free, Eq.(21) can also be written as:

$$
\operatorname{curl} \kappa_{e}=-\operatorname{curl} \kappa_{p}=\boldsymbol{\theta} .
$$

Another consequence arising from the discontinuity of the elastic/plastic rotation is that the elastic/plastic distortion tensors cannot be defined anymore. Only the elastic/plastic strain tensors remain single-valued and can be defined. Hence, the incompatibility equation (15) has now to be written as [deWit,1970]:

$$
\begin{aligned}
& \operatorname{curl} \boldsymbol{\epsilon}_{e}=+\boldsymbol{\alpha}+\boldsymbol{\kappa}_{e}^{t}-\operatorname{tr}\left(\boldsymbol{\kappa}_{e}\right) \mathbf{I} \\
& \operatorname{curl} \boldsymbol{\epsilon}_{p}=-\boldsymbol{\alpha}+\boldsymbol{\kappa}_{p}^{t}-\operatorname{tr}\left(\boldsymbol{\kappa}_{p}\right) \mathbf{I}
\end{aligned}
$$

The discontinuity of the elastic rotation due to the presence of disclinations is the Frank vector and reads:

$$
\Omega=\int_{C} \boldsymbol{\kappa}_{e} \cdot \mathbf{d r}
$$

The discontinuity of the elastic displacement due to dislocations is the Burgers vector $\mathbf{b}$ : 


$$
\mathbf{b}=\int_{C}\left(\boldsymbol{\epsilon}_{e}-\left(\boldsymbol{\kappa}_{e}^{t} \times \mathbf{r}\right)^{t}\right) \cdot \mathbf{d r} \mathbf{r}
$$

where $\mathbf{r}$ is a position vector. Note that $\mathbf{b}$ contains a nonlocal strain contribution stemming from the non-uniformity of elastic curvatures in the defects core [deWit,1970]. Nevertheless, like the Frank vector $\boldsymbol{\Omega}, \mathbf{b}$ is a point-wise measure of lattice incompatibility. If $S$ is the surface of unit normal $\mathbf{n}$ delimited by the circuit $C$, using Stokes' theorem allows rewriting Eqs. $(25,26)$ as:

$$
\begin{aligned}
\boldsymbol{\Omega} & =\int_{S} \boldsymbol{\theta} \cdot \mathbf{n} d S \\
\mathbf{b} & =\int_{S}\left(\boldsymbol{\alpha}-\left(\boldsymbol{\theta}^{t} \times \mathbf{r}\right)^{t}\right) \cdot \mathbf{n} d S .
\end{aligned}
$$

In contrast with the Burgers and Frank vectors, the disclination and dislocation densities $\boldsymbol{\theta}$ and $\boldsymbol{\alpha}$ are continuous tensorial renditions of incompatibility. They provide a natural regularization of the singular and discontinuous nature of the Frank/Burgers vectors. The incompatibility tensor $\boldsymbol{\eta}$ defined as

$$
\boldsymbol{\eta}=\operatorname{curl} \operatorname{curl}^{t} \epsilon_{e}-\operatorname{curl} \kappa_{e}
$$

is a combined measure of the incompatibility arising from both dislocations and disclinations. Using Eqs.(22,23), it can be rewritten as 


$$
\boldsymbol{\eta}=\operatorname{curl}\left(\frac{1}{2} \operatorname{tr}(\boldsymbol{\alpha}) \mathbf{I}-\boldsymbol{\alpha}^{t}\right)-\boldsymbol{\theta}
$$

in terms of the dislocation and disclination density tensors.

\section{Nonlocal elastic laws}

We assume a scalar-valued free energy density functional at any material point $\mathbf{r}$ in the body $V$ in the following integral form :

$$
\begin{aligned}
\psi(\mathbf{r}) & =\frac{1}{2} C_{i j k l}(\mathbf{r}) \epsilon_{i j}^{e}(\mathbf{r}) \epsilon_{k l}^{e}(\mathbf{r})+\frac{1}{2} A_{i j k l}(\mathbf{r}) \kappa_{i j}^{e}(\mathbf{r}) \kappa_{k l}^{e}(\mathbf{r})+\ldots \\
\ldots & +\frac{1}{2} \epsilon_{i j}^{e}(\mathbf{r}) \int_{V} D_{i j k l}\left(\mathbf{r}-\mathbf{r}^{\prime}\right) \epsilon_{k l}^{e}\left(\mathbf{r}^{\prime}\right) d V^{\prime}+\ldots \\
\ldots & +\frac{1}{2} \kappa_{i j}^{e}(\mathbf{r}) \int_{V} B_{i j k l}\left(\mathbf{r}-\mathbf{r}^{\prime}\right) \kappa_{k l}^{e}\left(\mathbf{r}^{\prime}\right) d V^{\prime}
\end{aligned}
$$

The first two pointwise terms on the r.h.s. involve the symmetric positivedefinite quadratic forms $\mathbf{A}$ and $\mathbf{C}$ given at location $\mathbf{r}$. They lead to the linear elastic constitutive laws used and discussed in [deWit,1970, Fressengeas,2011, Upadhyay,2013] in the framework of field models for dislocation and disclina-

tion fields. Nonlinearity of elasticity may well play a role in the core regions of the defects, but it will not be considered here. The novelty in this work is the introduction of nonlocal contributions to the constitutive equations, in the form of the Eringen-type nonlocal convolution integrals appearing in the last two terms of the r.h.s. These terms systematize earlier attempts at describing the elastic response of defects cores [Taupin,2014]. They contain 
convolution products between the fourth-order elasticity tensors $\mathbf{B}$ and $\mathbf{D}$, and the elastic curvature and strain tensors respectively. In these convolution integrals, $\mathbf{r}^{\prime}$ denotes any material point distant from point $\mathbf{r}$. We also define the vector $\mathbf{R}^{\prime}=\left(\mathbf{r}^{\prime}-\mathbf{r}\right)$ with $\mathbf{R}^{\prime} \cdot \mathbf{R}^{\prime}=R^{\prime 2}$. The norms of $\mathbf{B}$ and $\mathbf{D}$ must decay rapidly to zero with increasing relative distance $R^{\prime}$ [Eringen,2002]. The elastic moduli tensors $(\mathbf{C}, \mathbf{D})$ are in units of $M P a$, while $(\mathbf{A}, \mathbf{B})$ have dimension MPa.m ${ }^{2}$. As such, (A, B) involve an internal length scale and possess nonlocal character. Due to the symmetry of $\boldsymbol{\epsilon}_{e}$ and to their quadratic character, $\mathbf{A}$ and $\mathbf{C}$ have the symmetries:

$$
\begin{aligned}
& A_{i j k l}=A_{k l i j} \\
& C_{i j k l}=C_{j i k l}=C_{i j l k}=C_{k l i j}
\end{aligned}
$$

However, $\mathbf{B}$ and $\mathbf{D}$ do not need to present the same symmetries as $\mathbf{A}$ and $\mathbf{C}$ respectively, as shall be discussed below. We may write $\psi$ in a more convenient form as follows:

$$
\begin{aligned}
\psi(\mathbf{r}) & =\frac{1}{2} C_{i j k l}(\mathbf{r}) \epsilon_{i j}^{e}(\mathbf{r}) \epsilon_{k l}^{e}(\mathbf{r})+\frac{1}{2} A_{i j k l}(\mathbf{r}) \kappa_{i j}^{e}(\mathbf{r}) \kappa_{k l}^{e}(\mathbf{r})+\ldots \\
\ldots & +\frac{1}{2} \epsilon_{i j}^{e}(\mathbf{r}) \int_{V} \mathcal{K}^{\alpha}\left(\mathbf{R}^{\prime}\right) D_{i j k l}\left(\mathbf{r}, \mathbf{r}^{\prime}\right) \epsilon_{k l}^{e}\left(\mathbf{r}^{\prime}\right) d V^{\prime}+\ldots \\
\ldots & +\frac{1}{2} \kappa_{i j}^{e}(\mathbf{r}) \int_{V} \mathcal{K}^{\theta}\left(\mathbf{R}^{\prime}\right) B_{i j k l}\left(\mathbf{r}, \mathbf{r}^{\prime}\right) \kappa_{k l}^{e}\left(\mathbf{r}^{\prime}\right) d V^{\prime}
\end{aligned}
$$

In this relation, $\mathcal{K}^{\alpha}\left(\mathbf{R}^{\prime}\right)$ and $\mathcal{K}^{\theta}\left(\mathbf{R}^{\prime}\right)$ denote nonlocal kernels, while the elastic 
tensors $\mathbf{B}$ and $\mathbf{D}$ are now defined at locations $\mathbf{r}^{\prime}$. The superscript $\alpha$ (resp. $\left.\theta\right)$ is used to suggest that the range of the associated nonlocal kernel corresponds to the spatial extent of the dislocation (resp. disclination) core. However, we will further assume for the sake of simplicity that $\mathcal{K}^{\alpha}\left(\mathbf{R}^{\prime}\right)=\mathcal{K}^{\theta}\left(\mathbf{R}^{\prime}\right)=$ $\mathcal{K}\left(\mathbf{R}^{\prime}\right)$ in the following. We have in mind that the convolution integrals are associated with a defected region of the crystal containing dislocations and/or disclinations, and that the kernel range should therefore be the order of the characteristic length of this area. As already suggested, we also expect that nonlocal elasticity ceases to be significant in the perfectly ordered regions of the crystal, where only conventional elasticity laws should apply. Nonlocality arises from the coupling between the strongly varying strain and curvature fields at locations $\mathbf{r}$ and $\mathbf{r}^{\prime}$ in crystal defect cores. Indeed, the nonlocal displacement $\mathbf{d} \mathbf{u}$ and strain $\boldsymbol{\epsilon}_{e}$ at location $\mathbf{r}^{\prime}$ deriving from the rotation $\mathbf{d} \boldsymbol{\omega}_{e}$ generated by the curvature $\boldsymbol{\kappa}_{e}$ at location $\mathbf{r}$ are (see illustration in Fig.1.A):

$$
\mathbf{d u}=\mathbf{d} \boldsymbol{\omega}_{e} \times \mathbf{R}^{\prime}=\boldsymbol{\kappa}_{e}(\mathbf{r}) \cdot \mathbf{d r} \times \mathbf{R}^{\prime}=\boldsymbol{\epsilon}_{e}\left(\mathbf{r}^{\prime}\right) \cdot \mathbf{d} \mathbf{r}^{\prime}=\boldsymbol{\epsilon}_{e}\left(\mathbf{r}^{\prime}\right) \cdot(-\mathbf{d r}),
$$

or in component form:

$$
d u_{k}=e_{k m n} d \omega_{m}^{e} R_{n}^{\prime}=e_{k m n} \kappa_{m l}^{e}(\mathbf{r}) d x_{l} R_{n}^{\prime}=-\epsilon_{k l}^{e}\left(\mathbf{r}^{\prime}\right) d x_{l},
$$

a relation from which we extract the strain tensor induced at location $\mathbf{r}^{\prime}$ by 

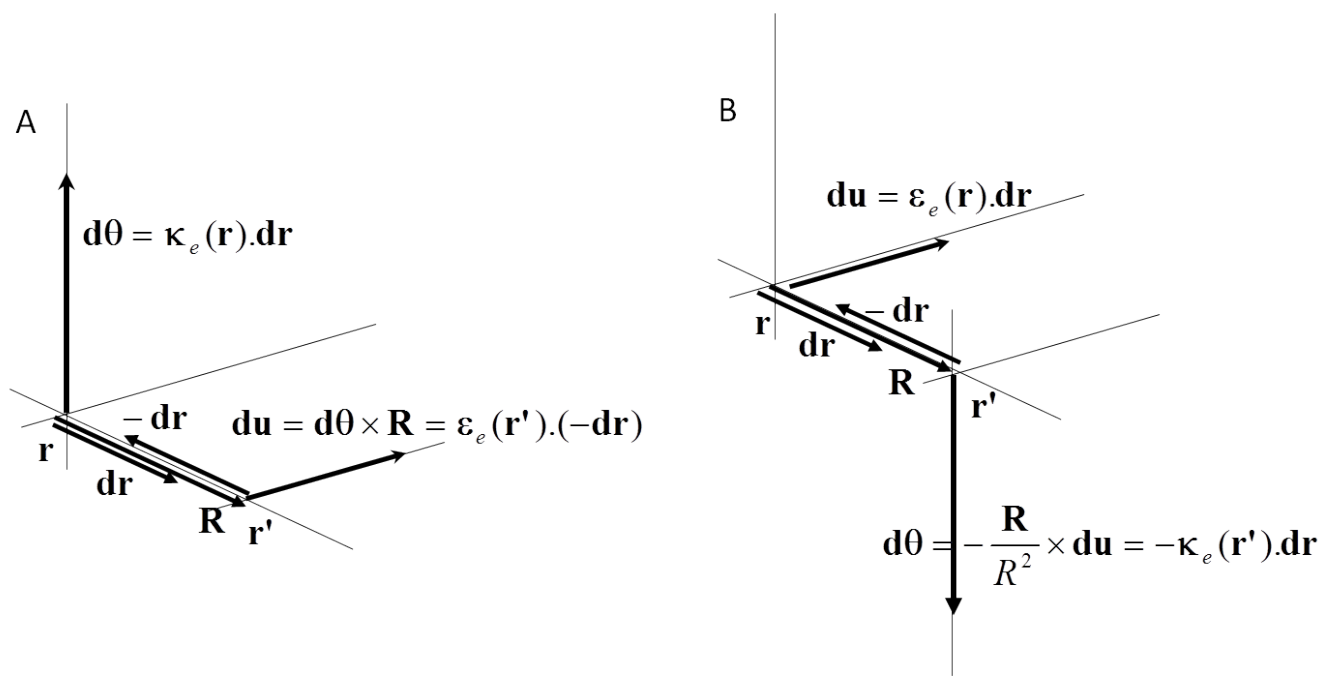

Figure 1: Nonlocal strains and curvatures. A) Nonlocal shear strain at location $\mathbf{r}^{\prime}$ induced by the rotation $\mathbf{d} \boldsymbol{\theta}$ arising from a curvature tensor at location $\mathbf{r}$; B) Nonlocal rotation and curvature at location $\mathbf{r}^{\prime}$ from the displacement $\mathbf{d u}$ induced by a shear strain tensor at location $\mathbf{r}$.

the curvature tensor at location $\mathbf{r}$ :

$$
\epsilon_{k l}^{e}\left(\mathbf{r}^{\prime}\right)=-e_{k m n} \kappa_{m l}^{e}(\mathbf{r}) R_{n}^{\prime}, \quad \epsilon_{e}\left(\mathbf{r}^{\prime}\right)=-\left(\boldsymbol{\kappa}_{e}^{t}(\mathbf{r}) \times \mathbf{R}^{\prime}\right)^{t} .
$$

Note the presence of this curvature-induced strain tensor in the definition of the Burgers vector in Eq.(26) above.

Similarly, the rotation $\mathbf{d} \boldsymbol{\omega}_{e}$ and curvature $\boldsymbol{\kappa}_{e}$ at location $\mathbf{r}^{\prime}$ stemming from the displacement $\mathbf{d} \mathbf{u}$ induced by the strain $\boldsymbol{\epsilon}_{e}$ at location $\mathbf{r}$ (see Fig.1.B) are:

$$
\mathbf{d} \boldsymbol{\omega}_{e}=\frac{-\mathbf{R}^{\prime}}{R^{\prime 2}} \times \mathbf{d u}=\frac{-\mathbf{R}^{\prime}}{R^{2}} \times \boldsymbol{\epsilon}_{e}(\mathbf{r}) \cdot \mathbf{d r}=\boldsymbol{\kappa}_{e}\left(\mathbf{r}^{\prime}\right) \cdot \mathbf{d} \mathbf{r}^{\prime}=-\boldsymbol{\kappa}_{e}\left(\mathbf{r}^{\prime}\right) \cdot \mathrm{dr} \mathbf{r}
$$


In component form, we obtain:

$$
d \omega_{k}^{e}=e_{k m n} d u_{m} \frac{R_{n}^{\prime}}{R^{\prime 2}}=e_{k m n} \epsilon_{m l}^{e}(\mathbf{r}) \frac{R_{n}^{\prime}}{R^{\prime 2}} d x_{l}=-\kappa_{k l}^{e}\left(\mathbf{r}^{\prime}\right) d x_{l}
$$

from which we find the curvature tensor at location $\mathbf{r}^{\prime}$ stemming from the strain tensor at location $\mathbf{r}$ :

$$
\kappa_{k l}^{e}\left(\mathbf{r}^{\prime}\right)=-e_{k m n} \epsilon_{m l}^{e}(\mathbf{r}) \frac{R_{n}^{\prime}}{R^{\prime 2}}, \quad \boldsymbol{\kappa}_{e}\left(\mathbf{r}^{\prime}\right)=-\frac{1}{R^{\prime 2}}\left(\boldsymbol{\epsilon}_{e}(\mathbf{r}) \times \mathbf{R}^{\prime}\right)^{t}
$$

Now, consider the third term $\psi_{3}$ in Eq.34. Using Eq.37, it reads

$$
\psi_{3}(\mathbf{r})=-\frac{1}{2} \epsilon_{i j}^{e}(\mathbf{r}) \int_{V} \mathcal{K}\left(\mathbf{R}^{\prime}\right) D_{i j k l}\left(\mathbf{r}, \mathbf{r}^{\prime}\right) e_{k m n} \kappa_{m l}^{e}(\mathbf{r}) R_{n}^{\prime} d V^{\prime}
$$

The curvature tensor at location $\mathbf{r}$ can be extracted from the integral:

$$
\psi_{3}(\mathbf{r})=-\frac{1}{2} \epsilon_{i j}^{e}(\mathbf{r})\left(\int_{V} \mathcal{K}\left(\mathbf{R}^{\prime}\right) e_{k m n} D_{i j k l}\left(\mathbf{r}, \mathbf{r}^{\prime}\right) R_{n}^{\prime} d V^{\prime}\right) \kappa_{m l}^{e}(\mathbf{r})
$$

and a coupling tensor $\bar{D}_{i j m l}(\mathbf{r})$ can be defined at location $\mathbf{r}$ :

$$
\bar{D}_{i j m l}(\mathbf{r})=-\int_{V} \mathcal{K}\left(\mathbf{R}^{\prime}\right) e_{k m n} D_{i j k l}\left(\mathbf{r}^{\prime}\right) R_{n}^{\prime} d V^{\prime}
$$

such that:

$$
\psi_{3}(\mathbf{r})=\frac{1}{2} \epsilon_{i j}^{e}(\mathbf{r}) \bar{D}_{i j m l}(\mathbf{r}) \kappa_{m l}^{e}(\mathbf{r})
$$


Changing the subscript labels, we obtain:

$$
\begin{aligned}
\bar{D}_{i j k l}(\mathbf{r}) & =\int_{V} \mathcal{K}\left(\mathbf{R}^{\prime}\right) e_{k m n} D_{i j m l}\left(\mathbf{r}, \mathbf{r}^{\prime}\right) R_{n}^{\prime} d V^{\prime} \\
\psi_{3}(\mathbf{r}) & =\frac{1}{2} \epsilon_{i j}^{e}(\mathbf{r}) \bar{D}_{i j k l}(\mathbf{r}) \kappa_{k l}^{e}(\mathbf{r}),
\end{aligned}
$$

and we note that $\overline{\mathbf{D}}(\mathbf{r})$ can be written as

$$
\overline{\mathbf{D}}(\mathbf{r})=\int_{V} \mathcal{K}\left(\mathbf{R}^{\prime}\right)\left(\mathbf{D}^{t} \times \mathbf{R}^{\prime}\right)^{t} d V^{\prime}
$$

where it is understood that transposing affects the last two subscripts of a fourth order tensor. Similarly, consider the fourth term $\psi_{4}$ in Eq.34. Using Eq.40, it reads

$$
\begin{aligned}
& \psi_{4}(\mathbf{r})=-\frac{1}{2} \kappa_{i j}^{e}(\mathbf{r}) \int_{V} \mathcal{K}\left(\mathbf{R}^{\prime}\right) B_{i j k l}\left(\mathbf{r}, \mathbf{r}^{\prime}\right) e_{k m n} \epsilon_{m l}^{e}(\mathbf{r}) \frac{R_{n}^{\prime}}{R^{\prime 2}} d V^{\prime} \\
& \psi_{4}(\mathbf{r})=-\frac{1}{2} \kappa_{i j}^{e}(\mathbf{r})\left(\int_{V} \mathcal{K}\left(\mathbf{R}^{\prime}\right) e_{k m n} B_{i j k l}\left(\mathbf{r}, \mathbf{r}^{\prime}\right) \frac{R_{n}^{\prime}}{R^{\prime 2}} d V^{\prime}\right) \epsilon_{m l}^{e}(\mathbf{r})
\end{aligned}
$$

Define $\bar{B}_{i j m l}(\mathbf{r})$

$$
\bar{B}_{i j m l}(\mathbf{r})=-\int_{V} \mathcal{K}\left(\mathbf{R}^{\prime}\right) e_{k m n} B_{i j k l}\left(\mathbf{r}, \mathbf{r}^{\prime}\right) \frac{R_{n}^{\prime}}{R^{\prime 2}} d V^{\prime}
$$

such that

$$
\psi_{4}(\mathbf{r})=\frac{1}{2} \kappa_{i j}^{e}(\mathbf{r}) \bar{B}_{i j m l}(\mathbf{r}) \epsilon_{m l}^{e}(\mathbf{r}),
$$


and change the subscript labels to obtain:

$$
\begin{aligned}
\bar{B}_{i j k l}(\mathbf{r}) & =\int_{V} \mathcal{K}\left(\mathbf{R}^{\prime}\right) e_{k m n} B_{i j m l}\left(\mathbf{r}, \mathbf{r}^{\prime}\right) \frac{R_{n}^{\prime}}{R^{\prime 2}} d V^{\prime} \\
\psi_{4}(\mathbf{r}) & =\frac{1}{2} \kappa_{i j}^{e}(\mathbf{r}) \bar{B}_{i j k l}(\mathbf{r}) \epsilon_{k l}^{e}(\mathbf{r}) .
\end{aligned}
$$

Note that $\overline{\mathbf{B}}(\mathbf{r})$ can be written as

$$
\overline{\mathbf{B}}(\mathbf{r})=\int_{V} \mathcal{K}\left(\mathbf{R}^{\prime}\right)\left(\mathbf{B} \times \frac{\mathbf{R}^{\prime}}{R^{\prime 2}}\right)^{t} d V^{\prime}
$$

As a consequence of Eqs. $(34,46,53)$, all tensors $(\mathbf{A}, \mathbf{C}, \overline{\mathbf{B}}, \overline{\mathbf{D}})$ in the free energy density functional are now evaluated at location $\mathbf{r}$ :

$$
\psi=\frac{1}{2} C_{i j k l} \epsilon_{i j}^{e} \epsilon_{k l}^{e}+\frac{1}{2} A_{i j k l} \kappa_{i j}^{e} \kappa_{k l}^{e}+\frac{1}{2} \bar{D}_{i j k l} \epsilon_{i j}^{e} \kappa_{k l}^{e}+\frac{1}{2} \bar{B}_{i j k l} \kappa_{i j}^{e} \epsilon_{k l}^{e} .
$$

Hence, the energy functional is now defined pointwise but it contains elastic cross-moduli tensors $\overline{\mathbf{B}}(\mathbf{r})$ and $\overline{\mathbf{D}}(\mathbf{r})$ that are nonlocal in nature. Note that, unlike the $\mathbf{B}$ and $\mathbf{D}$ pointwise cross-moduli, the convoluted cross-moduli $(\overline{\mathbf{B}}, \overline{\mathbf{D}})$ have same dimension and are in units of MPa.m. Through derivations with respect to the elastic strains and curvatures, the work conjugates are obtained as:

$$
\begin{aligned}
T_{i j}^{s y m} & =\frac{\partial \psi}{\partial \epsilon_{i j}^{e}}=C_{i j k l} \epsilon_{k l}^{e}+\frac{1}{2}\left(\bar{D}_{i j k l}+\bar{B}_{k l i j}\right) \kappa_{k l}^{e} \\
M_{i j}^{d e v} & =\frac{\partial \psi}{\partial \kappa_{i j}^{e}}=A_{i j k l} \kappa_{k l}^{e}+\frac{1}{2}\left(\bar{B}_{i j k l}+\bar{D}_{k l i j}\right) \epsilon_{k l}^{e} .
\end{aligned}
$$


By defining the tensor $\bar{E}_{i j k l}=\frac{1}{2}\left(\bar{D}_{i j k l}+\bar{B}_{k l i j}\right)$, the elastic constitutive laws read finally:

$$
\begin{aligned}
T_{i j}^{s y m} & =\frac{\partial \psi}{\partial \epsilon_{i j}^{e}}=C_{i j k l} \epsilon_{k l}^{e}+\bar{E}_{i j k l} \kappa_{k l}^{e} \\
M_{i j}^{d e v} & =\frac{\partial \psi}{\partial \kappa_{i j}^{e}}=A_{i j k l} \kappa_{k l}^{e}+\bar{E}_{k l i j} \epsilon_{k l}^{e} .
\end{aligned}
$$

We thus retrieve relationships formally similar to those proposed in previous work [Upadhyay,2013], except that the elastic cross-moduli tensors $(\overline{\mathbf{B}}, \overline{\mathbf{D}}, \overline{\mathbf{E}})$ now have integral form. Note that, due to the symmetry of $\boldsymbol{\epsilon}_{e},(\overline{\mathbf{B}}, \overline{\mathbf{D}}, \overline{\mathbf{E}})$ have the following symmetries:

$$
\begin{aligned}
& \bar{B}_{i j k l}=\bar{B}_{i j l k} \\
& \bar{D}_{i j k l}=\bar{D}_{j i k l} \\
& \bar{E}_{i j k l}=\bar{E}_{j i k l}
\end{aligned}
$$

The next section now aims at proposing admissible forms and expressions for the tensors $(\overline{\mathbf{B}}, \overline{\mathbf{D}})$ defined in Eqs. $(47,54)$.

\section{Admissible forms of nonlocal elasticity tensors}

As already mentioned, it is desired that the convoluted cross-moduli tensors $(\overline{\mathbf{B}}, \overline{\mathbf{D}})$ be non-zero in the defected areas and vanish in the perfect crystal, and that the range of nonlocality be on the order of the characteristic length of the defect core. Further, $(\overline{\mathbf{B}}, \overline{\mathbf{D}})$ must satisfy the symmetry conditions $(60,61)$, 
and definite-positiveness of the elastic energy density functional should be ensured for some kernel and some convolution domain. Let us first consider tensor $\overline{\mathbf{D}}$. As aforementioned, the cross-moduli tensor $\mathbf{D}$ does not need to have the same form and symmetries as $\mathbf{C}$. However, as proposed earlier by Eringen [Eringen,1977a, Eringen,1977b], we shall assume $\mathbf{D}\left(\mathbf{r}^{\prime}\right)$ in the form

$$
\mathbf{D}\left(\mathbf{r}^{\prime}\right)=\phi\left(\mathbf{r}^{\prime}\right) \mathbf{C}
$$

where $\phi\left(\mathbf{r}^{\prime}\right)$ is a scalar function to be determined in the following and the elastic moduli tensor $\mathbf{C}$ is constant. It can easily be checked that $\overline{\mathbf{D}}(\mathbf{r})$ then satisfies the symmetry conditions (61). Choosing similarly the tensor $\mathbf{B}\left(\mathbf{r}^{\prime}\right)$ in the form of $\mathbf{A}\left(\mathbf{r}^{\prime}\right)$ and using the isotropic expression of tensor $\mathbf{A}$ proposed in [Upadhyay,2013] yields a convoluted tensor $\overline{\mathbf{B}}$ that violates the symmetry condition (60). Thus, assuming that $\overline{\mathbf{D}}$ is known from the above, we choose instead to determine $\overline{\mathbf{B}}$ by imposing the symmetry relations

$$
\bar{B}_{i j k l}=\bar{D}_{k l i j},
$$

which allows to satisfy the symmetry conditions (60). Using this assumption, the last two terms of the free energy density $\psi$ in Eq.55 are now formally 
identical, and the constitutive equations read:

$$
\begin{aligned}
T_{i j}^{s y m} & =\frac{\partial \psi}{\partial \epsilon_{i j}^{e}}=C_{i j k l} \epsilon_{k l}^{e}+\bar{D}_{i j k l} \kappa_{k l}^{e} \\
M_{i j}^{d e v} & =\frac{\partial \psi}{\partial \kappa_{i j}^{e}}=A_{i j k l} \kappa_{k l}^{e}+\bar{D}_{k l i j} \epsilon_{k l}^{e} .
\end{aligned}
$$

From Eqs. $(45,52,64)$, the symmetry conditions $(64)$ are effective when

$$
\int_{V} \mathcal{K}\left(\mathbf{R}^{\prime}\right) e_{k m n} B_{i j m l}\left(\mathbf{r}^{\prime}\right) \frac{R_{n}^{\prime}}{R^{\prime 2}} d V^{\prime}=\int_{V} \mathcal{K}\left(\mathbf{R}^{\prime}\right) e_{i m n} D_{k l m j}\left(\mathbf{r}^{\prime}\right) R_{n}^{\prime} d V^{\prime}
$$

or equivalently

$$
\int_{V} \mathcal{K}\left(\mathbf{R}^{\prime}\right)\left(e_{k m n} \frac{B_{i j m l}\left(\mathbf{r}^{\prime}\right)}{R^{\prime 2}}-e_{i m n} D_{k l m j}\left(\mathbf{r}^{\prime}\right)\right) R_{n}^{\prime} d V^{\prime}=0 .
$$

Because $e_{k m n} e_{i m n}=\delta_{i k}$, a sufficient condition for Eq.68 to hold is:

$$
\forall \mathbf{r}^{\prime}, \frac{B_{k j m l}\left(\mathbf{r}^{\prime}\right)}{R^{\prime 2}}-D_{k l m j}\left(\mathbf{r}^{\prime}\right)=0
$$

or, changing the subscripts:

$$
\forall \mathbf{r}^{\prime}, B_{i j k l}\left(\mathbf{r}^{\prime}\right)=R^{\prime 2} D_{i l k j}\left(\mathbf{r}^{\prime}\right)
$$

Eq.70 appropriately removes the $1 / R^{\prime 2}$ singularity in the definition (54) of tensor $\overline{\mathbf{B}}$, and shows clearly that the symmetry (64) postulated at integral level does not apply locally at points $\mathbf{r}^{\prime}$. We note however that Eq.70 cannot 
be used to constrain non-vanishing cross-moduli $B_{i j k l}\left(\mathbf{r}^{\prime}\right)$ if the integration domain $V$ is shrinked down to the point-wise limit by making $R^{\prime} \rightarrow 0$. In this case, the singularity derails the attempt at satisfying the symmetry relations (64) at convoluted level. Consequences on the stability of balanced elastic fields will be examined in the next Section. We now focus on proposing an admissible form of tensor $\mathbf{D}\left(\mathbf{r}^{\prime}\right)$ in the regular situation where the integration domain is kept finite. The definition of the convoluted tensor $\overline{\mathbf{D}}$ in Eq.(47) involves a cross product $\mathbf{D} \times \mathbf{R}^{\prime}$. Hence, if $\phi\left(\mathbf{r}^{\prime}\right)$ is taken as a spatially homogeneous function and if the integration volume $V$ is centrosymmetric, a condition that we can arbitrarily fulfill, then relation (47) is also centrosymmetric and $\overline{\mathbf{D}}$ vanishes. As already discussed, this is consistent with the work of [Upadhyay,2013] showing that the elastic cross-moduli tensors are null in centrosymmetric conditions and isotropic elasticity. In the presence of dislocations and disclinations however, centrosymmetry is broken in the defects core, and $\overline{\mathbf{D}}$ becomes non-zero. To ensure that such behavior is limited to the core area, we chose $\phi\left(\mathbf{r}^{\prime}\right)$ so as to reflect the defect densities at point $\mathbf{r}^{\prime}$. We take it to be non-zero in the presence of defects and zero in their absence by simply using the following form:

$$
\phi\left(\mathbf{r}^{\prime}\right)=\frac{\left\|\boldsymbol{\eta}\left(\mathbf{r}^{\prime}\right)\right\|}{\eta_{0}}
$$

where $\|\boldsymbol{\eta}\|$ is the norm of the incompatibility tensor introduced in Eqs. $(29,30)$, and $\eta_{0}$ a reference incompatibility value. 


\section{Positive-definiteness of the free energy density}

To ensure stability of the the elastic strain and curvature fields, we now look for sufficient conditions ensuring that the functional $\psi$ be positive-definite at any point, for any strain and curvature field, appropriate kernel functions and finite integration domains $V$. Let us define for convenience the state vector $\mathbf{X}$ containing the six independent elastic strain components and the nine deviatoric elastic curvature components in the following order:

$$
\mathbf{X}^{t}=\left(\epsilon_{11}^{e} \epsilon_{22}^{e} \epsilon_{33}^{e} 2 \epsilon_{32}^{e} 2 \epsilon_{31}^{e} 2 \epsilon_{12}^{e} \kappa_{11}^{e} \kappa_{12}^{e} \kappa_{13}^{e} \kappa_{21}^{e} \kappa_{22}^{e} \kappa_{23}^{e} \kappa_{31}^{e} \kappa_{32}^{e} \kappa_{33}^{e}\right)
$$

Let also $\mathrm{Q}$ be the $15 \times 15$ matrix containing all elastic moduli in the block form:

$$
\mathbf{Q}=\left(\left(\begin{array}{ccc}
C_{1111} & \cdots & C_{1112} \\
\vdots & \ddots & \vdots \\
C_{1211} & \cdots & C_{1212}
\end{array}\right)\left(\begin{array}{ccc}
\bar{D}_{1111} & \cdots & \bar{D}_{1133} \\
\vdots & \ddots & \vdots \\
\bar{D}_{1211} & \cdots & \bar{D}_{1233}
\end{array}\right)\right)
$$

such that the free energy density at a point $\mathbf{r}$ in the body reads in the synthetic form

$$
\psi(\mathbf{r})=\frac{1}{2} \mathbf{X}(\mathbf{r})^{t} \cdot \mathbf{Q}(\mathbf{r}) \cdot \mathbf{X}(\mathbf{r})
$$


As a consequence of Sylvester's theorem, the functional $\psi$ is symmetric positive-definite if all principal minors of matrix $\mathbf{Q}$ are positive [Born,1954]. Hence, we now look for conditions between the elastic moduli deriving from this requirement, at any point $\mathbf{r}$ in the material, for any non-zero elastic strain and curvature field. For the sake of simplicity, we assume isotropic elastic moduli $C_{i j k l}$ :

$$
C_{i j k l}=\lambda \delta_{i j} \delta_{k l}+\mu\left(\delta_{i k} \delta_{j l}+\delta_{i l} \delta_{j k}\right)
$$

The $A_{i j k l}$ moduli are taken in the simple following form [Upadhyay,2013]:

$$
A_{i j k l}=\mu l_{\kappa}^{2} \delta_{i k} \delta_{j l}
$$

where $l_{\kappa}$ is a characteristic arm's-length, usually taken as a fraction of the Burgers vector length [Fressengeas,2014, Seif,2014]. The tensor $\overline{\mathbf{D}}$ is defined by Eqs. $(47,63,71)$. It can be rewritten in the form:

$$
\bar{D}_{i j m l}(\mathbf{r})=-e_{k m n} \int_{V} \mathcal{K}\left(\mathbf{R}^{\prime}\right) \frac{\left\|\boldsymbol{\eta}\left(\mathbf{r}^{\prime}\right)\right\|}{\eta_{0}} R_{n}^{\prime} d V^{\prime} C_{i j k l}=-e_{k m n} l_{n}(\mathbf{r}) C_{i j k l}
$$

In the above, $l_{n}(\mathbf{r})=\int_{V} \mathcal{K}\left(\mathbf{R}^{\prime}\right) \frac{\left\|\boldsymbol{\eta}\left(\mathbf{r}^{\prime}\right)\right\|}{\eta_{0}} R_{n}^{\prime} d V^{\prime}$ is a length defining the range of nonlocality of the convoluted cross-moduli in direction $\mathbf{e}_{n} . l_{n}(\mathbf{r})$ does not define a spatially homogenous field, as its local (signed) value depends on the defect density fields and on the nonlocal kernel. As aforementioned, $l_{n}(\mathbf{r})$ and 
the convoluted tensors $\overline{\mathbf{B}}$ and $\overline{\mathbf{D}}$ vanish in the absence of defects. Let us define the characteristic nonlocal length $l_{N L}$ as $l_{N L}=\sqrt{l_{n} l_{n}}$. Simple closed-form expressions of the principal minors of the matrix $\mathbf{Q}$ can be obtained if all non zero coefficients $D_{i j k l}$ are taken to be equal to $\mu$. In this case, positivedefiniteness of the free energy density functional at point $\mathbf{r}$ is ensured if the following conditions are satisfied:

$$
\begin{aligned}
& l_{\kappa}^{2}>0 \\
& l_{\kappa}^{2}>4 l_{N L}^{2} \\
& l_{\kappa}^{2}>3 l_{N L}^{2} \\
& l_{\kappa}^{2}>5 l_{N L}^{2} \\
& l_{\kappa}^{2}>2 l_{N L}^{2} \\
& l_{\kappa}^{2}>\frac{2 \lambda+10 \mu}{\lambda+2 \mu} l_{N L}^{2} \\
& l_{\kappa}^{2}>\frac{2 \lambda+8 \mu}{\lambda+\mu} l_{N L}^{2} \\
& l_{\kappa}^{2}>\frac{6 \lambda+22 \mu}{3 \lambda+2 \mu} l_{N L}^{2} .
\end{aligned}
$$

With the elastic moduli $(\lambda, \mu)$ of copper used in the forthcoming simulations and listed in Table 1, the most constraining of the above inequalities is Eq.81. By defining the ratio $r_{N L}=l_{N L} / l_{\kappa}$, Eq.81 implies $r_{N L}<1 / \sqrt{5}(\approx 0.45)$. Hence, the nonlocal arm's-length $l_{\kappa}$ of tensor A being a fraction of the Burgers vector magnitude, the nonlocality range $l_{N L}$ of the cross-moduli tensors 
$(\overline{\mathbf{B}}, \overline{\mathbf{D}})$ should be limited to the core region of defects, consistent with our prescriptions. In all forthcoming simulations, the components $D_{i j k l}$ are actually taken according to Eq.63, not all equal to $\mu$ as in the above simplified derivation. In that case, such a derivation becomes extremely intricate, but it can be found numerically that the energy density functional is positive everywhere if $r_{N L}<0.22$, which also implies that the range of nonlocality is strictly limited to the core of the defects. Positive-definiteness of the free energy functional cannot be guaranteed if the integration domain $V$ is driven down to the point-wise limit, because the symmetry (64) of the crossmoduli at convoluted level does not hold anymore, as shown above. As a consequence, instability of the elastic strain and curvature fields should be expected. Similar conclusions were recently reached in the development of point-wise first order strain gradient elastic constitutive models for dislocation cores [Admal,2016]. In this work, several point-wise free energy density functionals were built, but most of them were indefinite, implying instability of the elastic fields. Atomistic calculations then suggested that an integral positive-definiteness criterion would warrant stability of these fields. In the present paper, this result is reached within the continuum model, thanks to the introduction of nonlocal convolution terms in the constitutive formulations. 


\section{FFT spectral approximation of higher order balance equations}

Owing to the complexity of the elasto-static equations of field dislocation and disclination mechanics involving convolution integrals in their constitutive relationships, solutions of boundary value problems are now looked for in periodic media using a spectral method with a Fast Fourier Transform algorithm, due to numerical efficiency of the latter. Note however, that they could also have been found by using variational formulations and extended finite element techniques [Polizzotto,2001]. Here, a recently developed iterative fixed-point algorithm generalizing earlier work by [Moulinec,1998] to dislocation and generalized disclination fields in elastically heterogeneous materials [Berbenni,2014] will be extended, in particular to deal with convo-

lution integrals. Although the elastic convoluted cross-moduli tensors $\overline{\mathbf{B}}(\mathbf{r})$ and $\overline{\mathbf{D}}(\mathbf{r})$ are nonlocal, the elastic laws $(56,57)$ are formulated point-wise. Thus, they formally appear as a local elastic framework with heterogeneous moduli tensors. Of course, although such will not be the case in this work, the tensors $\mathbf{A}$ and $\mathbf{C}$ could be heterogeneous as well. Hence, the moduli tensors can be written in the form

$$
\begin{aligned}
& \mathbf{C}(\mathbf{r})=\mathbf{C}^{0}+\delta \mathbf{C}(\mathbf{r}), \\
& \mathbf{A}(\mathbf{r})=\mathbf{A}^{0}+\delta \mathbf{A}(\mathbf{r}), \\
& \overline{\mathbf{E}}(\mathbf{r})=\overline{\mathbf{E}}^{0}+\delta \overline{\mathbf{E}}(\mathbf{r}),
\end{aligned}
$$


where the homogeneous tensors $\mathbf{C}^{\mathbf{0}}, \mathbf{A}^{\mathbf{0}}$ and $\overline{\mathbf{E}}^{\mathbf{0}}$ involve moduli for a reference medium, and $(\boldsymbol{\delta} \mathbf{C}(\mathbf{r}), \boldsymbol{\delta} \mathbf{C}(\mathbf{r}), \boldsymbol{\delta} \overline{\mathrm{E}}(\mathbf{r}))$ denote heterogeneous fluctuations. In the numerical scheme to be presented below, $\mathbf{C}^{\mathbf{0}}, \mathbf{A}^{\mathbf{0}}$ and $\overline{\mathbf{E}}^{\mathbf{0}}$ will be taken as the volume average of the corresponding tensors. As will be evidenced in the simulation results, the volume average of the nonlocal cross-moduli is zero, and we thus skip $\overline{\mathbf{E}}^{\mathbf{0}}$ in the following. Consequently, we now reformulate the stress and couple stress tensors defined in Eqs. $(56,57)$ as

$$
\begin{aligned}
& T_{i j}^{s y m}=C_{i j k l}^{0} \epsilon_{k l}^{e \|}+C_{i j k l} \epsilon_{k l}^{e \perp}+\delta C_{i j k l} \epsilon_{k l}^{e \|}+\delta \bar{E}_{i j k l}\left(\kappa_{k l}^{e \perp}+\kappa_{k l}^{e \|}\right) \\
& M_{i j}^{d e v}=A_{i j k l}^{0} \kappa_{k l}^{e \|}+A_{i j k l} \kappa_{k l}^{e \perp}+\delta A_{i j k l} \kappa_{k l}^{e \|}+\delta \bar{E}_{k l i j}\left(\epsilon_{k l}^{e \perp}+\epsilon_{k l}^{e \|}\right),
\end{aligned}
$$

and we define the polarization tensors

$$
\begin{aligned}
\tau_{i j}^{\|} & =\delta C_{i j k l} \epsilon_{k l}^{e \|}+\delta \bar{E}_{i j k l} \kappa_{k l}^{e \|} \\
\tau_{i j}^{\perp} & =C_{i j k l} \epsilon_{k l}^{e \perp}+\delta \bar{E}_{i j k l} \kappa_{k l}^{e \perp} \\
\phi_{i j}^{\|} & =\delta A_{i j k l} \kappa_{k l}^{e \|}+\delta \bar{E}_{k l i j} \epsilon_{k l}^{e \|} \\
\phi_{i j}^{\perp} & =A_{i j k l} \kappa_{k l}^{e \perp}+\delta \bar{E}_{k l i j} \epsilon_{k l}^{e \perp},
\end{aligned}
$$

such that the stress and couple stress tensors finally read 


$$
\begin{aligned}
& T_{i j}^{s y m}=C_{i j k l}^{0} \epsilon_{k l}^{e \|}+\tau_{i j}^{\|}+\tau_{i j}^{\perp} \\
& M_{i j}^{d e v}=A_{i j k l}^{0} \kappa_{k l}^{e \|}+\phi_{i j}^{\|}+\phi_{i j}^{\perp} .
\end{aligned}
$$

In the above, $\tau_{i j}^{\|}$and $\phi_{i j}^{\|}$are respectively compatible stress and couple stress polarization tensors associated solely with heterogeneous elasticity, while $\tau_{i j}^{\perp}$ and $\phi_{i j}^{\perp}$ are respectively incompatible stress and couple stress polarization tensors associated with the presence of defects. The latter disappear in the absence of incompatibility. The stress and couple stress tensors defined in Eqs. $(95,96)$ satisfy the higher order balance equation [Mindlin,1962]

$$
\operatorname{div}\left(\mathbf{T}^{\mathrm{sym}}\right)+\frac{1}{2} \operatorname{curl}\left(\operatorname{div} \mathbf{M}^{\mathrm{dev}}\right)=0
$$

which reads in component form

$$
T_{i j, j}^{s y m}+\frac{1}{2} e_{i k l} M_{l m, m k}^{d e v}=0 .
$$

We now switch from the real space to the Fourier space. Let $\hat{\lambda}$ denote the Fourier transform of a function $\lambda$ and $\boldsymbol{\xi}$ be the Fourier frequency vector with components $\xi_{i}$ in a reference cartesian frame. The imaginary number is denoted by $i$. The balance equation (98) becomes

$$
i \xi_{j} \hat{T}_{i j}^{s y m}-\frac{1}{2} e_{i k l} \xi_{m} \xi_{k} \hat{M}_{l m}^{d e v}=0
$$


By using the expressions of stresses and couple stresses in Eqs. $(95,96)$, as well as the compatibility relations $U_{i j}^{e \|}=u_{i, j}$ and $\kappa_{i j}^{e \|}=1 / 2 e_{i k l} u_{l, k j}$, Eq.(99) yields

$$
\begin{aligned}
& \left(\xi_{l} \xi_{j} C_{i j k l}^{0}-\frac{1}{4} e_{i j l} A_{l m p q}^{0} e_{p r k} \xi_{m} \xi_{j} \xi_{r} \xi_{q}\right) \hat{u}_{k} \\
& \quad=i \xi_{j}\left(\hat{\tau}_{i j}^{\|}+\hat{\tau}_{i j}^{\perp}\right)-\frac{1}{2} e_{i j l} \xi_{m} \xi_{j}\left(\hat{\phi}_{l m}^{\|}+\hat{\phi}_{l m}^{\perp}\right) .
\end{aligned}
$$

The above equations set an algebraic linear system of the form

$$
\hat{G}_{i k} \hat{u}_{k}=\hat{f}_{i}
$$

where $\hat{\mathbf{G}}$ is associated with the homogeneous reference moduli tensors $\mathbf{C}_{\mathbf{0}}$ and $\mathbf{A}_{\mathbf{0}}$, and $\hat{u}_{k}$ is the unknown displacement. The latter is given by

$$
\hat{u}_{k}=\hat{G}_{k i}^{-1} \hat{f}_{i}
$$

Eq.102 is implicit because the unknown displacement is contained in the compatible stress and couple stress polarization tensors in $\hat{f}_{i}$. From the expression of the displacements, we now derive the strain and curvature components. The strains are

$$
\hat{\epsilon}_{k l}^{e \|}=\frac{i}{2}\left(\hat{G}_{k i}^{-1} \xi_{l}+\hat{G}_{l i}^{-1} \xi_{k}\right) \hat{f}_{i}
$$


and the curvatures read

$$
\hat{\kappa}_{i j}^{e \|}=-\frac{1}{2} e_{i l k} \xi_{j} \xi_{l} \hat{u}_{k}=-\frac{1}{2} e_{i l k} \xi_{j} \xi_{l} \hat{G}_{k m}^{-1} \hat{f}_{m}
$$

We now cast the compatible and incompatible stress and couple stress polarization tensors in $\hat{\mathbf{f}}$. Eq.(103) becomes

$$
\begin{aligned}
\hat{\epsilon}_{k l}^{e \|}+\frac{1}{2}\left(\hat{G}_{k i}^{-1} \xi_{l}+\hat{G}_{l i}^{-1} \xi_{k}\right) & \left(\xi_{j} \hat{\tau}_{i j}^{\|}+\frac{i}{2} e_{i j l} \xi_{m} \xi_{j} \hat{\phi}_{l m}^{\|}\right) \\
= & -\frac{1}{2}\left(\hat{G}_{k i}^{-1} \xi_{l}+\hat{G}_{l i}^{-1} \xi_{k}\right)\left(\xi_{j} \hat{\tau}_{i j}^{\perp}+\frac{i}{2} e_{i j l} \xi_{m} \xi_{j} \hat{\phi}_{l m}^{\perp}\right),
\end{aligned}
$$

and Eq.(104) is transformed into

$$
\begin{aligned}
\hat{\kappa}_{i j}^{e \|}+\frac{1}{2} e_{i l k} \xi_{j} \xi_{l} \hat{G}_{k m}^{-1}\left(i \xi_{n} \hat{\tau}_{m n}^{\|}-\frac{1}{2} e_{m n p} \xi_{n} \xi_{q} \hat{\phi}_{p q}^{\|}\right) \\
\quad=-\frac{1}{2} e_{i l k} \xi_{j} \xi_{l} \hat{G}_{k m}^{-1}\left(i \xi_{n} \hat{\tau}_{m n}^{\perp}-\frac{1}{2} e_{m n p} \xi_{n} \xi_{q} \hat{\phi}_{p q}^{\perp}\right) .
\end{aligned}
$$

By defining the following fourth-order tensors: 


$$
\begin{aligned}
\hat{\Gamma}_{k l i j}^{0} & =\frac{1}{2}\left(\hat{G}_{k i}^{-1} \xi_{l}+\hat{G}_{l i}^{-1} \xi_{k}\right) \xi_{j}, \\
\hat{\Gamma}_{k l m n}^{1} & =\frac{i}{4}\left(\hat{G}_{k i}^{-1} \xi_{l}+\hat{G}_{l i}^{-1} \xi_{k}\right) e_{i j m} \xi_{n} \xi_{j}, \\
\hat{\Gamma}_{i j m n}^{2} & =\frac{i}{2} e_{i l k} \xi_{j} \xi_{l} \hat{G}_{k m}^{-1} \xi_{n}, \\
\hat{\Gamma}_{i j p q}^{3} & =-\frac{1}{4} e_{i l k} \xi_{j} \xi_{l} \hat{G}_{k m}^{-1} e_{m n p} \xi_{n} \xi_{q}
\end{aligned}
$$

Eqs. $(105,106)$ are of the form

$$
\begin{aligned}
& \hat{\boldsymbol{\epsilon}}^{e \|}+\hat{\boldsymbol{\Gamma}}^{0}: \hat{\boldsymbol{\tau}}^{\|}+\hat{\boldsymbol{\Gamma}}^{1}: \hat{\boldsymbol{\phi}}^{\|}=-\hat{\boldsymbol{\Gamma}}^{0}: \hat{\boldsymbol{\tau}}^{\perp}-\hat{\boldsymbol{\Gamma}}^{1}: \hat{\boldsymbol{\phi}}^{\perp} \\
& \hat{\boldsymbol{\kappa}}^{e \|}+\hat{\boldsymbol{\Gamma}}^{2}: \hat{\boldsymbol{\tau}}^{\|}+\hat{\boldsymbol{\Gamma}}^{3}: \hat{\boldsymbol{\phi}}^{\|}=-\hat{\boldsymbol{\Gamma}}^{2}: \hat{\boldsymbol{\tau}}^{\perp}-\hat{\boldsymbol{\Gamma}}^{3}: \hat{\boldsymbol{\phi}}^{\perp} .
\end{aligned}
$$

Coming back from the Fourier space to the real space and substituting the compatible and incompatible stress and couple stress polarization tensors for their expressions (91-94), Eq.(111) becomes

$$
\begin{aligned}
\boldsymbol{\epsilon}^{e \|} & +\Gamma^{0} *\left(\boldsymbol{\delta} \mathbf{C}: \boldsymbol{\epsilon}^{e \|}+\delta \overline{\mathbf{E}}: \boldsymbol{\kappa}^{e \|}\right)+\Gamma^{1} *\left(\boldsymbol{\delta} \mathbf{A}: \boldsymbol{\kappa}^{e \|}+\delta \overline{\mathbf{E}}^{t}: \boldsymbol{\epsilon}^{e \|}\right) \\
& =\left\langle\boldsymbol{\epsilon}^{e \|}\right\rangle-\Gamma^{0} *\left(\mathbf{C}: \boldsymbol{\epsilon}^{e \perp}+\delta \overline{\mathbf{E}}: \boldsymbol{\kappa}^{e \perp}\right)-\Gamma^{1} *\left(\mathbf{A}: \boldsymbol{\kappa}^{e \perp}+\delta \overline{\mathbf{E}}^{t}: \boldsymbol{\epsilon}^{e \perp}\right),
\end{aligned}
$$

and Eq.(112) finally reads 


$$
\begin{aligned}
& \boldsymbol{\kappa}^{e \|}+\Gamma^{2} *\left(\boldsymbol{\delta} \mathbf{C}: \boldsymbol{\epsilon}^{e \|}+\delta \overline{\mathbf{E}}: \boldsymbol{\kappa}^{e \|}\right)+\Gamma^{3} *\left(\boldsymbol{\delta} \mathbf{A}: \boldsymbol{\kappa}^{e \|}+\delta \overline{\mathbf{E}}^{t}: \boldsymbol{\epsilon}^{e \|}\right) \\
& \quad=\left\langle\boldsymbol{\kappa}^{e \|}\right\rangle-\Gamma^{2} *\left(\mathbf{C}: \boldsymbol{\epsilon}^{e \perp}+\delta \overline{\mathbf{E}}: \boldsymbol{\kappa}^{e \perp}\right)-\Gamma^{3} *\left(\mathbf{A}: \boldsymbol{\kappa}^{e \perp}+\delta \overline{\mathbf{E}}^{t}: \boldsymbol{\epsilon}^{e \perp}\right),
\end{aligned}
$$

where $\left\langle\boldsymbol{\epsilon}^{e \|}\right\rangle$ and $\left\langle\boldsymbol{\kappa}^{e \|}\right\rangle$ are volume averages of strain and curvature tensors, respectively. Note that, for convenience, transposing in Eqs.(113,114) has the meaning: $\bar{E}_{i j k l}^{t}=\bar{E}_{k l i j}$, which is different from the transpose operation used in Eqs. $(47,54)$.

Eqs. $(113,114)$ constitute an implicit coupled set of linear algebraic equations for the unknown compatible elastic strain and curvature tensors. The solution algorithm is provided below. The reader is referred to [Djaka,2016] for a complete description of such an algorithm and for details on numerical aspects. Note however that the solution of Poisson-type equations for the determination of the incompatible elastic strain and curvature tensors in the Fourier space uses differentiation rules based on centered finite differences for the calculation of spatial derivatives [Berbenni,2014], while for all other spatial derivatives in the algorithm, in particular those involved in the fourth-order tensors defined in Eqs.(107-110), the classical differentiation rule is used. 


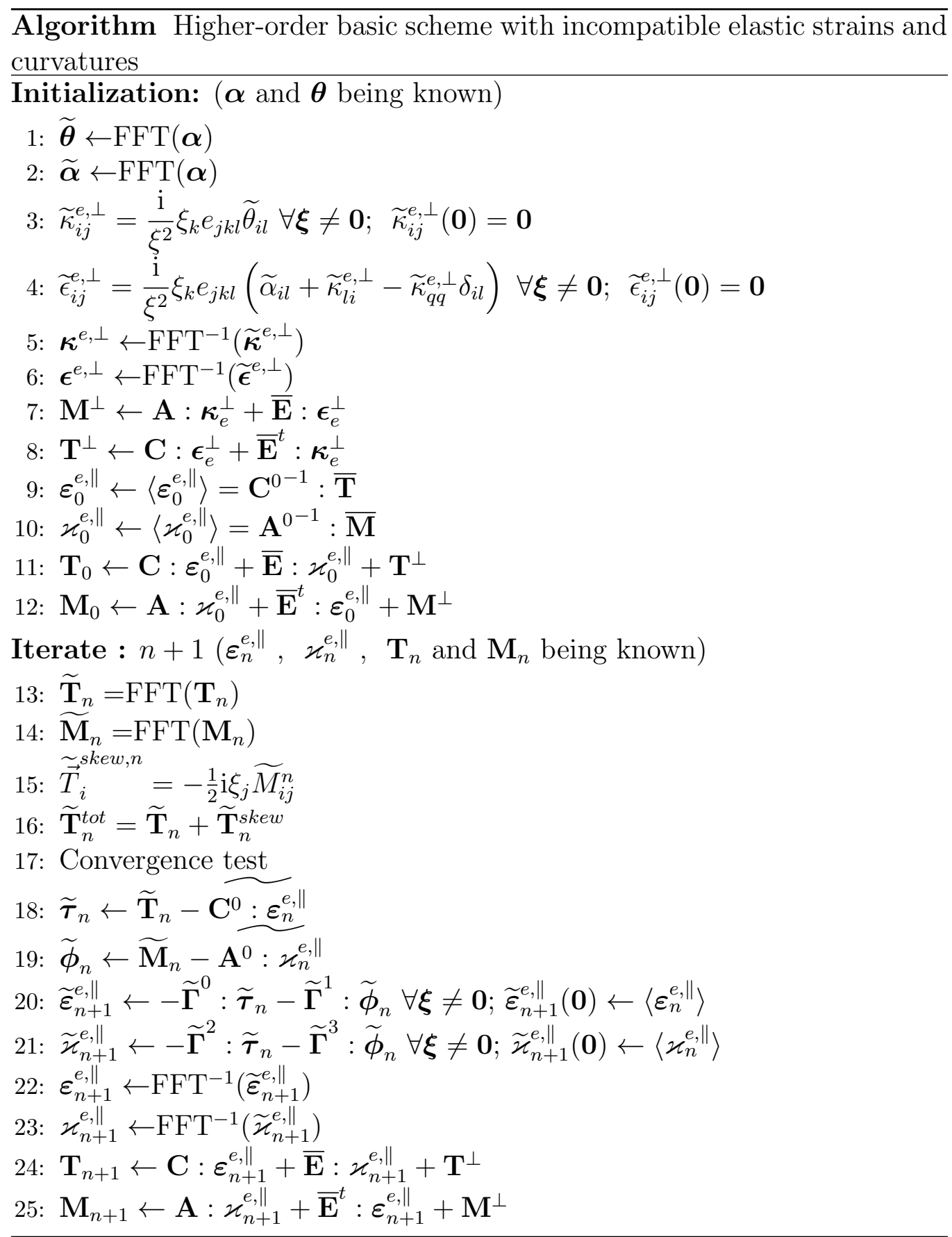




\section{Applications: single edge/screw dislocations, wedge dislocation dipoles and tilt boundaries}

In this Section, we intend to show the effects the nonlocal stress and couple stress fields derived from the above convolution scheme on the defects cores and their stability. While the model and numerical code are fully threedimensional, we shall limit ourselves to two-dimensional configurations. Of course, as we use a spectral method with FFT scheme, the boundary conditions will be periodic in all directions. We shall consider straight edge and screw dislocations, with dislocation lines parallel to the unit vector $\mathbf{e}_{3}$. The Burgers vector of the edge dislocations lies in the plane $\left(\mathbf{e}_{1}, \mathbf{e}_{2}\right)$ normal to $\mathbf{e}_{\mathbf{3}}$. Thus, edge dislocations with Burgers vector parallel to the direction $\mathbf{e}_{\mathbf{1}}$

(resp. $\mathbf{e}_{2}$ ) are rendered by the density $\alpha_{13}$ (resp. $\alpha_{23}$ ). The Burgers vector of the screw dislocations is parallel to $\mathbf{e}_{\mathbf{3}}$ and the dislocations are therefore described by the density $\alpha_{33}$. We shall further consider wedge disclinations with disclination line and Frank vector parallel to $\mathbf{e}_{\mathbf{3}}$, and described by the density $\theta_{33}$. The material is copper and the material/numerical parameters are listed in Table 1 . The grid size is $\delta=0.025 b$, where $b$ is the Burgers vector magnitude, which is sufficiently small to ensure a proper continuous description of elastic fields within the core region of defects. Disclination and dislocation cores are introduced in the grid by using Gaussian distributions of the associated defect densities. The latter are spread on cylinders of radius $r_{0}=b$, and the Gaussian standard deviation is $\sigma_{0}=0.1 b$. For the sake of simplicity, the nonlocal kernel $\mathcal{K}\left(\mathbf{R}^{\prime}\right)$ is taken to be spatially isotropic. We 
Table 1: Simulation parameters.

\begin{tabular}{ccccc}
$b$ & $\mu$ & $\nu$ & $l^{\kappa}$ & $r_{N L}$ \\
\hline $4.04 \AA$ & $23 G P a$ & 0.3647 & $0.5 \AA$ & 0.21 \\
\hline$\delta$ & $r_{0}$ & $\sigma_{0}$ & $\sigma_{N L}$ & \\
\hline $0.025 b$ & $b$ & $0.1 b$ & $0.18 b$ &
\end{tabular}

use the following three-dimensional normalised Gaussian function:

$$
\mathcal{K}\left(\mathbf{R}^{\prime}\right)=\frac{1}{\sigma_{N L}^{3}(2 \pi)^{3 / 2}} \exp \left(-\frac{\mathbf{R}^{\prime 2}}{2 \sigma_{N L}^{2}}\right)
$$

with standard deviation $\sigma_{N L}=0.18 b$. Anisotropic functions may be envisioned in future work (see [Lazar,2011]). The ratio $r_{N L}$ introduced in Section 6 is taken to be $r_{N L}=0.21$, close to the critical value for positive-definiteness of the elastic energy. We have checked that the effects of nonlocal elasticity are less significant for smaller $r_{N L}$ values. The appropriate ratio is obtained by conveniently choosing the reference parameter for incompatibility $\eta_{0}$ in Eq. (71). In all simulations presented below, the iterative algorithm converges within $\approx 10$ iterations, with a convergence criterion similar to that adopted in [Djaka,2016]. No Gibbs oscillations were ever observed in the elastic fields.

In the following, the stress tensor $\mathbf{C}: \boldsymbol{\epsilon}^{\mathbf{e}}$ will be referred to as the "local stress", $\overline{\mathbf{E}}: \boldsymbol{\kappa}^{e}$ as the "nonlocal stress", the couple stress $\mathbf{A}: \boldsymbol{\kappa}^{\mathbf{e}}$ as the "local couple stress" and $\overline{\mathbf{E}}^{t}: \boldsymbol{\epsilon}^{e}$ as the "nonlocal couple stress". Although 


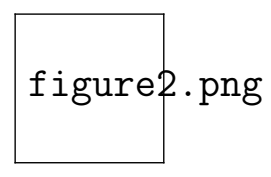

Figure 2: Nonlocal elastic cross-moduli in a single edge dislocation $\alpha_{13}$ and an equivalent wedge disclination dipole $\theta_{33}$. (a) Edge dislocation density field. (b) Component of the nonlocal tensor $\overline{\mathbf{D}}$ corresponding to the edge dislocation. (c) Wedge disclination density field. (d) Component of the nonlocal tensor $\overline{\mathbf{B}}$ corresponding to the wedge disclination dipole.

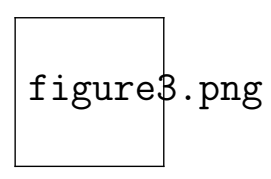

Figure 3: Single edge dislocation $\alpha_{13}$. (a) edge dislocation density field. (b) total in-plane symmetric shear stress field $T_{12}$. (c) nonlocal tensile stress field. (d) nonlocal in-plane symmetric shear stress field.

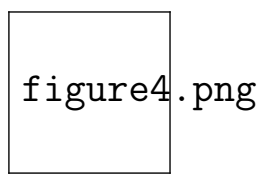

Figure 4: Wedge disclination dipole $\theta_{33}$. (a) wedge disclination density field. (b,c) Couple stress field in local (b) and nonlocal (c) elasticity. (d,e) Driving force on disclination density in local (d) and nonlocal (e) elasticity. (f,g) Energy density field in local (f) and nonlocal (g) elasticity. The same color scale is used in comparing the fields in local and nonlocal elasticity. 


\section{figure5.png}

Figure 5: Wedge disclination dipole $\theta_{33}$. Profiles of the couple stress field shown in Fig.(4) along the vertical line crossing the wedge disclination dipole. Green line with cross markers: total couple stress using local elasticity. Green line: total couple stress using nonlocal elasticity, i.e. sum of the local (purple line) and nonlocal (orange line) couple stresses.

figure6.png

Figure 6: Wedge disclination dipole $\theta_{33}$. Profiles of the driving force shown in Fig.(4) along the vertical line crossing the wedge disclination dipole. Green line with cross markers: local elasticity solution. Green line: nonlocal elasticity solution.

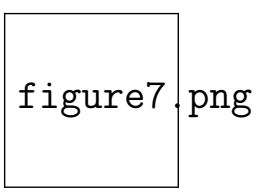

Figure 7: Wedge disclination dipole $\theta_{33}$. (a) wedge disclination density field. (b,c) In-plane symmetric shear stress field using local (b) and nonlocal (c) elasticity. (d,e) Tensile stress field $T_{11}$ using local (d) and nonlocal (e) elasticity. (f,g) Tensile stress field $T_{22}$ using local (f) and nonlocal (g) elasticity. The same color scale is used in comparing fields in local and nonlocal elasticity. 


\section{figure8.png}

Figure 8: Misfit profiles in wedge disclination dipole $\theta_{33}$ along horizontal midline. The curves show the misfit in the discrete Volterra dislocation model (blue), the standard Peierls-Nabarro model (green) and three variants of the present disclination-based model using more or less compact disclination cores, all other parameters being kept constant. Insets: The arrows represent the driving forces acting on (a) compact cores, (b) dilute cores. Diverging arrows in (a) suggest core spreading, converging arrows in (b) suggest core shrinking, pointing to stability of a relaxed equilibrium configuration.

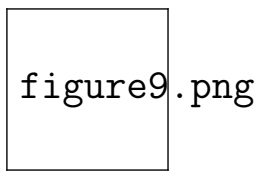

Figure 9: Close-up showing a cylindrical nanograin embedded in a matrix. The white dotted lines show the grain boundary and delimit the grain. (a) Wedge disclination dipoles $\theta_{33}$ forming the grain boundary. (b,c) driving forces on the wedge disclinations (arrows) in local (b) and nonlocal (c) elasticity superimposed on the wedge disclination density. (d,e) driving forces on the wedge disclinations (arrows) in local (d) and nonlocal (e) elasticity superimposed on the energy density. The red dotted lines in (c,e) suggest the evolution of the grain according to the driving forces. The same color scale is used in comparing fields in local and nonlocal elasticity. 


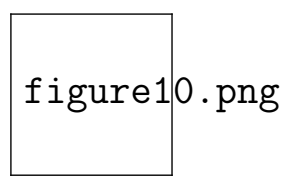

Figure 10: Interaction between the cylindrical grain shown in Fig.(9) and a screw dislocation $\alpha_{33}$ on the left hand side of the grain boundary $(\mathrm{a}, \mathrm{b})$ and entering the grain boundary $(\mathrm{c}, \mathrm{d})$. The white dotted lines show the grain boundary and delimit the grain. In (a,c), color-coded is shown the wedge disclination density field forming the grain boundary, while the arrows show the driving forces acting on them. In (b,d), color-coded is shown a nonlocal elastic modulus tensor $\bar{B}_{3112}$ due to both the wedge disclinations and the screw dislocation. The screw dislocation is represented by the circular spot in $(\mathrm{a}, \mathrm{c})$.

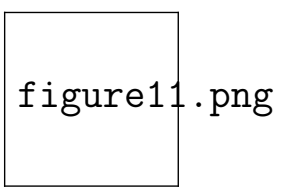

Figure 11: Elastic tilt induced in the core area of a tilt boundary in the presence of a screw dislocation and under applied external loading. The white dots underline the grain boundary. Color-coded is shown the elastic tilt (rotation about the axis normal to the figure). Blue and red spots indicate the location of the negative and positive wedge disclinations $\theta_{33}$ forming the grain boundary structure. In (a), the positive screw dislocation $\alpha_{33}$ lies at a distance on the left hand side of the boundary. The screw dislocation lies within the boundary in (b). In (c), a tensile stress $T_{11}$ of $1 G P a$ is applied normal to the boundary plane. In (d), an in-plane symmetric shear stress $T_{12}$ of $1 G P a$ is applied. 


\section{figure12.png}

Figure 12: Elastic rotation induced near a tilt boundary in the presence of a screw dislocation at different positions with respect to the grain boundary. In $(a, b, c)$ is shown the elastic rotation $\omega_{1}$. In $(\mathrm{d}, \mathrm{e}, \mathrm{f})$ is shown the elastic rotation $\omega_{2}$. In $(\mathrm{g}, \mathrm{h}, \mathrm{i})$ is shown the tilt elastic rotation $\omega_{3}$. The white dotted line underlines the grain boundary. Color-coded is shown the elastic rotation about the cartesian axis system. Blue and red spots indicate the location of the negative and positive wedge disclinations $\theta_{33}$ forming the grain boundary structure. The positive screw dislocation position is indicated by the white spot.

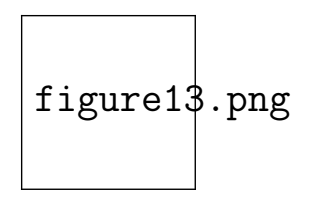

Figure 13: Different nanocrystalline configurations. The white dotted lines show the grain boundaries and delimit grains. Color-coded is shown the wedge disclination density $\theta_{33}$ forming the grain boundaries. The arrows indicate the driving forces acting on them. The red dotted lines suggest the eventual evolution of the grains according to the present driving forces. In (b), the nanocrystalline configuration is more even than in (a). In (c), an external in-plane shear stress of $1 \mathrm{GPa}$ is applied. In panel (d), four edge dislocations $\alpha_{13}$ (circular spots) are located at the centre of four grains. 
the present developments have elasto-static character, the driving forces for the motion of dislocation and disclination densities will be computed in order to suggest the eventual rearrangements of the defect density fields they potentially induce. Thus for instance, edge dislocations $\alpha_{13}$ should glide in direction $\mathbf{e}_{1}$ if driven by the Peach-Kehler force $F_{1}^{\alpha}=T_{12} \alpha_{13}$, and wedge disclinations $\theta_{33}$ should move in direction $\mathbf{e}_{1}$ (resp. $\mathbf{e}_{2}$ ) if driven by the PeachKoehler-type force $F_{1}^{\theta}=M_{32} \theta_{33}$ (resp. $\left.F_{2}^{\theta}=-M_{31} \theta_{33}\right)$. We recall that the mobility laws and transport equations controlling the motion of these defect densities were developed and solved in previous work of the present team [Fressengeas,2011, Fressengeas,2014]. The comprehensive study of the defects motion and subsequent plastic deformation will be the object of future work.

We first compare the cores of a single edge dislocation $\alpha_{13}$ and an "equivalent" wedge disclination dipole $\theta_{33}$ with small arm's-length, and their stability in their own stress and couple stress fields, at least tentatively, by checking the driving forces as indicated above. Here, "equivalent" means that both defects have the same Burgers vector and first-order stress field nearly coincident, even though they do not have the same Frank vector and the same couple stress field ${ }^{1}$. In the present configuration, the arm's-length of the disclination dipole is of the order of the Burgers vector, and the Frank vector length is the

\footnotetext{
${ }^{1}$ Here, the first order stress field is derived solely from the quadratic positive-definite contribution $\frac{1}{2} C_{i j k l} \epsilon_{i j}^{e} \epsilon_{k l}^{e}$ to the free energy.
} 
Burgers vector magnitude over the arm's-length (approximately $1 \mathrm{rad}$ ). These two configurations may be thought of as describing in two different ways the translational incompatibility and the core of the crystal defect, with the dislocation-based description overlooking the rotational incompatibility also involved in the defect. The procedure to check and possibly predict the stability of defect cores was the following. First, realistic Gaussian distributions of dislocation or disclination cores were arbitrarily fixed. Second, non-local elasticity parameters $\left(\sigma_{N L}, r_{N L}\right)$ were varied and their effect on elastic fields and core stability analysed. The standard deviation $\sigma_{N L}$ of the non-local kernel must be the order of a characteristic core size. The amplitude of non-local cross moduli can be varied through the term $r_{N L}$, with energy restrictions discussed in section 6 . The higher the value of $r_{N L}$, the higher the impact of non-local elasticity. It is instructive to discuss the forthcoming results in terms pertaining to the Peierls-Nabarro model [Peierls,1940, Nabarro,1947]. This model originally raised the issue of the equilibrium structure of an edge dislocation core, as described by a surface dislocation density distribution. As infinitesimal dislocation elements of the same sign tend to repel each other, the distribution was found to be in equilibrium only if its self-stress field was counter-balanced by a restoring stress field reflecting the resistance of the crystal to plastic slip. In order to obtain such a restoring stress field, the Peierls-Nabarro-type models introduce a complementary crystal misfit energy density depending on plastic slip, based either on phenomenological assumptions [Peierls,1940] or, more recently, on the generalized staking fault 
energy concept [Lu,2000, Shen,2004, Schoeck,2002, Gbemou,2016]. It is our intent to suggest that, in the present framework, restoring stresses and couple stresses may simply derive from the nonlocal convoluted part of the elastic free energy density, implying that no additional misfit energy is required.

Fig.2 shows the edge dislocation and wedge disclination density fields used in these descriptions, as well as the associated elastic cross-moduli. The figure suggests the following remarks: (i) the cross-moduli are non-zero in the core regions of the defects, and rapidly tend to zero as the current point moves away from the cores. (ii) for each isolated defect (either the dislocation or one single disclination), the cross-moduli are found in the form of dipoles. This feature derives from the cross-products involved in the definitions $(47,54)$ of the cross-moduli tensors. As a consequence, these moduli have null volume average. (iii) the cross-modulus $D_{1231}$ shown in panel (b) indicates that elastic curvatures $\kappa_{31}^{e}$ existing in the core area of the edge dislocation generate nonlocal shear stresses $T_{12}$, and $B_{3111}$ in panel (d) shows that tensile strains $\epsilon_{11}^{e}$ existing in the disclination cores generate nonlocal couple stresses $M_{31}$. Fig.3 describes the effects of nonlocality on the stress field in the edge dislocation area. Panel (b) shows the total shear stress field $T_{12}$. It is very similar to the conventional edge dislocation solution, except that it is non singular in the core area. Panel (d) shows the nonlocal part of this stress field. It can be seen that the latter counter-balances the local shear stress field in the core area, playing the role of a restoring stress field. However, the nonlocal 
shear stress magnitude is smaller than the local one by a factor 10, so that the total stress field is roughly similar to its local part. Panel (c) shows the nonlocal tensile stress $T_{11}$. Again, the latter opposes the local part of $T_{11}$ and plays the role of a restoring stress. However, since the nonlocal shear stress opposing the local shear stress it too low in magnitude, the edge dislocation distribution $\alpha_{13}$ is about to spread out under the action of the driving force $F_{1}^{\alpha}=T_{12} \alpha_{13}$, and stability of the defect is not expected. We have further checked that a more dilute or compact edge dislocation density is again not stable because the nonlocal stress field is always smaller than the local part.

In Figs.(4,5), we look similarly at the effects of nonlocality on the couple stress fields of the equivalent wedge disclination dipole $\theta_{33}$. Fig.4 compares the fields obtained in the point-wise case (left column) to those using the nonlocal convolution scheme (right column). Panels (b) and (c) show the couple stress field $M_{31}$. In the point-wise case, the earlier results of [Taupin,2013] are retrieved. The nonlocal convoluted couple stress field plays again the role of a restoring couple stress field, but here it exactly cancels the point-wise field. Therefore the couple stress field uniformly vanishes in the disclination core areas. Fig. 7 shows that the stress field of the wedge disclination dipole remains nearly unaffected by nonlocality, just like the edge dislocation stress field. Thus, the convolution terms have relatively little effect on the stress field of the crystal defect, whether in its dislocation-based or its disclinationbased "equivalent" descriptions, but they have strong effects on the cou- 
ple stress field in the disclination-based description. However, the driving forces $F_{1}^{\theta}=M_{32} \theta_{33}$ and $F_{2}^{\theta}=-M_{31} \theta_{33}$ acting on the wedge disclination density distribution do not involve the stress field, and they are cancelled by the counter-balancing action of the nonlocal convoluted couple stresses (see Figs. $(5,6))$. This property implies that a stable equilibrium configuration can be found for which no defect density spreading occurs. The misfit profiles obtained in this reference configuration and two other configurations with the same parameters, but with more compact or more extended disclination cores, are shown in Fig.8 and compared with the discontinuous Volterra misfit profile and the original Peierls-Nabarro misfit profile. It is clearly seen that the present disclination-based model has the ability to regularize the discontinuous misfit profile, and that it does not suffer from the well known inability of the Peierls-Nabarro-type solutions to reflect narrow cores [Joos,1994, Miller,1996]. Interestingly, the driving forces in the insets in Fig.8 show that the compact (respectively dilute) disclination cores will spread (respectively shrink), confirming that the reference configuration shown in Fig.4 is effectively stable. Note finally in panels (f) and (g) in Fig.4 that the point-wise and convoluted descriptions of the defect yield very similar energy density fields, because most of the energy is due to the first-order quadratic term $\frac{1}{2} C_{i j k l} \epsilon_{i j}^{e} \epsilon_{k l}^{e}$. The reason why the present framework allows predicting stable core configurations with disclination densities but not with dislocation densities is the following. As most of the elastic energy comes from the local term $\frac{1}{2} C_{i j k l} \epsilon_{i j}^{e} \epsilon_{k l}^{e}$, cancelling the dislocation density local stress field would 
require that the non-local energy contributions $\frac{1}{2} \bar{D}_{i j k l} \epsilon_{i j}^{e} \kappa_{k l}^{e}$ and $\frac{1}{2} \bar{B}_{i j k l} \kappa_{i j}^{e} \epsilon_{k l}^{e}$ have the same order of magnitudes, but with negative values. Within the energy positivity restrictions discussed in section 6 , it appears to be impossible to predict stability of dislocation densities. It might then be tempting to increase the value $l_{\kappa}$ in the local energy term $\frac{1}{2} A_{i j k l} \kappa_{i j}^{e} \kappa_{k l}^{e}$, as this quadratic term helps satisfying positivity of the energy and allows further increasing the contributions of non-local terms. However, couple stresses become high and the local stress field is increased due to mechanical balance, such that the global dislocation density energy becomes unrealistically high and still no stability is obtained. Concerning the disclination densities, stability is controlled by the energetic competition between the local term $\frac{1}{2} A_{i j k l} \kappa_{i j}^{e} \kappa_{k l}^{e}$ and the non-local terms. As the local term is smaller than the other term $\frac{1}{2} C_{i j k l} \epsilon_{i j}^{e} \epsilon_{k l}^{e}$, it is possible to ensure stability of disclination densities while easily respecting positivity of the total energy. We do not think that using other higher order elastic laws should allow stabilising dislocation densities (elastic strain gradients for instance, which are similar to elastic curvatures) because of the very important contribution of the quadratic term $\frac{1}{2} C_{i j k l} \epsilon_{i j}^{e} \epsilon_{k l}^{e}$. Using non-convex elastic potentials instead of simple linear elasticity might however be a good potential way to reduce the local stress field in the core of dislocations, such that non-local elasticity can have an impact and stability can be obtained.

The above results suggest that (i), it might be not necessary to introduce 
a complementary misfit energy to predict equilibrium defect structures, (ii) nonlocal convoluted elastic cross-moduli can yield stable defect structures without changing significantly the energy density field of the disclination dipole configuration and (iii), the disclination-based description of the translational crystal defect seems to capture more adequately its stable equilibrium structure than its dislocation-based representation. The latter point is further reinforced by the fact that the energy level of the wedge disclination dipole field is much lower than that of the equivalent edge dislocation field.

We now envision more complex defect configurations to further evidence the effects of nonlocal elasticity. We start with a single octogonal nanograin embedded in a matrix, as shown in the close-up in Fig.9. The grain is circled by a tilt boundary built from an array of wedge $\theta_{33}$ disclination dipoles and delineated by white dotted lines in the figure. The misorientation of the tilt boundary is approximately $30^{\circ}$. The left and right hand side columns of the figure compare the driving forces (the arrows show their orientation and magnitude) and the energy density fields in point-wise and nonlocal convoluted elasticity, respectively. Clearly, the energy density field is similar in both cases and most of the energy is located in the grain interior. However, the driving forces and disclination core interactions are radically different. In the point-wise elastic case, the self-couple stress field of the boundary yields a tendency at spreading the disclination cores, presumably until they are fully dispersed. Further, each disclination core feels only its own couple stress 
field, but not that of the neighboring cores, although the latter are extremely close. In the nonlocal convoluted elasticity simulation, no spreading trend can be detected in the driving force configuration, because the convoluted couple stress field counter-balances the local one. As a consequence, significant elastic interactions now take place between the neighboring cores. As suggested by the red dotted lines in the figure, the driving forces now induce refinement of the grain under the self elastic field of its boundary, leading to relaxation of the elastic energy stored in the grain. Of course, the kinetics of this grain-refinement trend are out of reach of the present elasto-static calculations, because it involves energy dissipation. We shall return to the issue in future work based on the present convoluted elastic constitutive relationships and the complete elasto-plastic framework of [Fressengeas,2011]. Fig.10 shows the same nanograin, complemented by an additional screw dislocation. In panel (a), the dislocation is located at a distance of the boundary, on its left hand side. It is assumed to be absorbed by the boundary, thus forming an extrinsic boundary dislocation located within a wedge disclination dipole in panel (c). Interactions of screw dislocations with tilt boundaries have been the obect of several investigations, including TEM observations [Priester,2001] and combined atomistic/discrete dislocation dynamic simulations [Dewald,2007], with rather disparate results on the absorption vs. transmission of impinging screws by the boundary. Continuous models have the potential for comprehensively exploring this field from a dynamic point of view. Here, we present only preliminary results in this perspective, being 
based on elasto-static simulations. Panels $(\mathrm{b}, \mathrm{d})$ show the relevant nonlocal elastic cross-modulus $\bar{B}_{3112}$ for both dislocation configurations. No elastic interaction between the screw dislocation and the tilt boundary is found if the nonlocal convoluted elastic moduli are cut off, whatever the location of the dislocation, because the elastic strain and curvature fields of the screw dislocation and wedge disclinations are strictly uncoupled. In contrast, interactions are found when the convoluted cross-moduli are activated and when the screw dislocation is absorbed by the boundary (see panel $(d)$ ), but not if it lies too far from the boundary as in panel (b). Panel (d) suggests indeed that the boundary interacts strongly with the screw dislocation. Fig.11 $(\mathrm{a}, \mathrm{b})$ illustrates further the accommodation of the screw dislocation by the boundary. Panel (a) shows the tilt rotation field when the screw dislocation is at a distance from the boundary. The rotation field of the boundary is nearly unaffected by the screw, and we note a symmetric pattern of alternating positive/negative tilt fluctuations accompanying the grain refinement process. In contrast, panel (b) clearly suggests that the absorption of the dislocation by the boundary strongly modifies the elastic rotation field. Indeed, the grain tends to be tilted quasi-uniformly counter-clockwise in the refinement process, as suggested by [Trautt,2012] using molecular dynamics simulations, while a clockwise tilt occurs in the adjacent matrix. The panels $(\mathrm{c}, \mathrm{d})$ show the structural changes arising from external loading of a flat tilt boundary, also made from an array of wedge disclination dipoles. Uniform $1 G P a$ tensile stress $T_{11}$ is applied in panel (c) and shear stress $T_{12}$ 
in panel (d). When cutting off the convoluted elastic cross-moduli in the simulations, no elastic tilt rotation field is observed. When they are turned on, it is seen in the figure that skew-symmetric tilt patterns are generated along the boundary in the tension case, whereas wavy tilt patterns occur in the in-plane shear case, so that the structure of the tilt boundary is significantly modified in both cases. Finally, in Fig. 12, we mimic again a screw dislocation approaching a tilt boundary that is now flat. The misorientation of the tilt boundary is approximately $30^{\circ}$. All elastic rotations $\omega_{1}, \omega_{2}$ and $\omega_{3}$ are shown in this figure. In the absence of the dislocation, the tilt boundary exhibits only symmetric fluctuations of the tilt rotation $\omega_{3}$. However, thanks to nonlocal effects, it can be seen that the presence of the dislocation induces significant twist rotations $\omega_{1}$ and $\omega_{2}$ in the grain boundary area. This suggests that complex dynamic interactions between the grain boundary and the dislocation will be obtained in future work when transport of defects and plasticity will be accounted for. As a last application, in Fig.13, a simple polycrystalline configuration is simulated by using the defect configuration shown in Fig. 9 and by taking advantage of the periodic boundary conditions (only the top left quarter of the panels is actually simulated). In panel (a), the periodic pattern comprises five octogonal grains, a larger one being at the center, and four rectangular grains. Panel (b) features a pattern with five identical octogonal grains and four square grains. Panel (c) shows the same configuration as panel (b), albeit with an applied in-plane shear stress of $1 G P a$. Finally, panel (d) shows the same configuration as panel (b), 
with an additional periodic pattern of four edge dislocations symmetrically located at the center of four octogonal grains. The driving forces acting on the grain boundary disclinations are shown in all panels. They suggest the eventual grain boundary motion, leading to grain growth or refinement. The differences in the panels suggest that the trend strongly depends on the con-

figuration of the polycrystal, as well as on the presence of external loading and extrinsic defects.

\section{Conclusions}

In this paper, we characterize the elastic response of dislocation and disclination cores in crystalline materials. Nonlinearity of elasticity may play a role at defect core level, but the present study only focuses on its nonlocal properties. Conventional pointwise elastic moduli tensors relating stresses to strains and couple-stresses to curvatures are adequately used to reflect the response of the defect-free regions of the crystals, because the lattice is centro-symmetric in these areas. However, centro-symmetry of the lattice is broken in the defected regions of the crystals, and elastic cross-moduli tensors relating curvatures to stresses and strains to couple-stresses need to be introduced in the constitutive relationships. In this aim, following [Eringen,2002], we use convolution integrals assigning at the current point weighted distributions of the stresses induced at distant points by the strong heterogeneity of the strain and curvature fields. The resulting convoluted elastic cross-moduli 
are defined at the current point as weighted distributions of the distant pointwise cross-moduli. Since it is the heterogeneity of the strain and curvature fields in the defects core regions that confers significance to the convoluted cross-moduli, the latter appear to be non-vanishing only in these regions. By construction, they are fully anisotropic. By assuming symmetry of the convoluted cross-moduli, sufficient conditions for positive-definiteness of the free energy can be obtained, and the stability of the solutions to boundary value elasticity problems can be ensured. However, these symmetry properties do not apply at point-wise level, implying that positive-definiteness of the free energy and stability of the elastic fields cannot be guaranteed in the limit of vanishingly small convolution domains (see also [Admal,2016]). When using finite integration domains, the convolution formalism allows reconciling non-convexity of the free energy at local level and stability of the solutions at integral level.

A spectral method using using a Fast Fourier Transform algorithm is introduced to solve the momentum and moment of momentum balance equations and to determine the stress and couple stress fields associated with the presence of prescribed dislocation/disclination density distributions in periodic media. Application of the method to the edge dislocation model and its "equivalent" wedge disclination dipoles representation suggests that, in the disclination-based interpretation, the convoluted cross-moduli provide the restoring "forces" needed to ensure compactness and stability of the defects 
core, simply from nonlocal convoluted elasticity. The couple stress field is instrumental in this feat, because it is involved in the driving forces on disclinations, and because it has intrinsically nonlocal character. Along with the compactness and stability of crystal defects, the introduction of the convoluted cross-moduli allows to account for their elastic interactions. For instance, grain refinement is obtained from the interactions of the disclination dipoles constitutive of a tilt boundary. Similarly, the accommodation of a screw dislocation as an extrinsic dislocation absorbed in a tilt boundary is investigated: a trend to quasi-uniform tilt of the grain is shown to accompany its refinement, as suggested by [Trautt,2012].

The above simulations are purely elasto-static, and calculations of the driving forces on defect densities were used to suggest the evolution of all envisionned configurations. However, the eventual kinetics of crystal defect distributions derive from dynamic dissipative mechanisms such as dislocations, disclination dipoles and grain boundaries motion, and cannot be determined from elasto-static theory. They will be investigated in future work by using the complete elasto-plastic version of the theory [Fressengeas,2014], as well as the recently developed FFT spectral method solving the defect transport equations [Djaka,2015, Djaka,2016]. The present results suggest that the introduction of nonlocal convoluted elasticity brings considerable predictive capabilities to the model regarding crystal defect stability, and grain boundary interactions with extrinsic defects. In particular, investigat- 
ing the effects of external loading on the grain boundary structure provides an avenue for the determination of the elastic cross-moduli [Seif,2014], with the help of atomistic simulations. In this feat, continuum metric methods [Zimmerman,2009, Tucker,2010, Tucker,2012, Tucker,2015, Sun,2016] will be used to prepare realistic initial defect density fields and to compare the predictions of continuum and atomistic simulations.

\section{Acknowledgements}

V. Taupin and C. Fressengeas would like to acknowledge the help of A. Villani, S. Berbenni and K.S. Djaka in the computational and numerical developments, and support by the French State through the National Research Agency under the program Investment in the future (Labex DAMAS referenced as ANR-11-LABX-0008-01) and Région Lorraine. L.Capolungo wishes to thank the office of Basic Energy Science Project E401 for it support.

\section{References}

\section{References}

[Acharya,2001] A. Acharya, J. Mech. Phys. Solids, 49, 761 (2001).

[Admal,2016] N.C. Admal, J. Marian, G. Po, J. Mech. Phys. Solids, In Press. 
[Berbenni,2014] S. Berbenni, V. Taupin, K.S. Djaka, C. Fressengeas, Int. J. Solids Structures 51, 4157 (2014).

[Born,1954] M. Born, K. Huang, in The International Series of Monographs on Physics, N.F. Mott et al. Ed., Oxford University Press, Amen House, London (1954).

[Cordier,2014] P. Cordier, S. Demouchy, B. Beausir, V. Taupin, F. Barou and C. Fressengeas, Nature 507, 51-56 (2014).

[Dewald,2007] M. P. Dewald and W. A. Curtin, Phil. Mag. 87, 4615-4641 (2007).

[deWit,1970] R. deWit, in Fundamental aspects of dislocation theory, ed. by J.A. Simmons, R. deWit and R. Bullough Nat. Bur. Stand. (US), Spec. Publ. 317, Vol. I, 651-673 (1970).

[Di Paola,2013] M. Di Paola, G. Failla, A. Pirrotta, A. Sofi, M. Zingales, Phil. Trans. R. Soc. A 371: 20120433 (2013).

[Dingreville,2014] R. Dingreville, A. Hallil, S. Berbenni, J. Mech. Phys. Solids 72, 40-60 (2014).

[Djaka,2015] K.S. Djaka, V. Taupin, S. Berbenni, C. Fressengeas, Modelling and Simulation in Materials Science and Engineering 23, 065008 (2015).

[Djaka,2016] K.S. Djaka, A. Villani, V. Taupin, L. Capolungo, S. Berbenni, Comp. Mater. Sc. (2016), submitted. 
[Eringen,1966] A.C. Eringen, Int. J. Engng. Sci. 4, 179-202 (1966).

[Eringen,1972] A.C. Eringen, D.G.B. Edelen, Int. J. Engng. Sci. 10, 233-248 (1972).

[Eringen,1977a] A.C. Eringen, B.S. Kim, Crystal Lattice Defects 7, 51-57 (1977).

[Eringen,1977b] A.C. Eringen, Technical Report No. 45, Civil Engng. Res. Report No. 77-SM-1, Princeton University (1977).

[Eringen,2002] A.C. Eringen, Non local continuum field theories, Springer, New York (2002).

[Feynman,1939] , R.P. Feynman, Ph.D. thesis, Massachusetts Institute of Technology (1939).

[Fressengeas,2011] C. Fressengeas, V. Taupin and L. Capolungo, Int. J. Solids Structures, 48, 3499-3509 (2011).

[Fressengeas,2014] C. Fressengeas, V. Taupin, L. Capolungo, Int. J. Solids Structures 51, 1434 (2014).

[Gbemou,2016] K. Gbemou, V. Taupin, J.M. Raulot, C. Fressengeas, Int. J. Plasticity 82, 241 (2016).

[Hardy,1982] R.J. Hardy, J. Chem. Phys. 76, 622-628 (1982). 
[Irvin-Kirkwood,1950] J.H. Irving, J.G. Kirkwood, J. Chem. Phys. 18, 817$829(1950)$.

[Joos,1994] B. Joós, Q. Ren, M.S. Duesberry, Phys. Rev. B 50, 5890 (1994).

[Kröner,1980] E. Kröner, in Physics of Defects, Ed. R. Balian et al., North Holland, Amsterdam, 218-314 (1980).

[Lazar,2003] M. Lazar, J. Physics A: Math. Gen. 36, 1415 (2003).

[Lazar,2011] M. Lazar, E. Agiasofitou, Int. J. Eng. Science 49, 1404-1414 (2011).

[Lazar,2015] M. Lazar, G. Po, Eur. J. Mech. A/Solids 50 ,152-162 (2015).

[Lu,2000] G. Lu, N. Kioussis, V.V. Bulatov, E. Kaxiras, Phys. Rev. B 62, 3099-3108 (2000).

[Miller,1996] R. Miller, R. Phillips, Philos. Mag. A 73, 803 (1996).

[Mindlin,1962] R.D. Mindlin, H.F. Tiersten, Arch. Rat. Mech. Anal., 11, 415 (1962).

[Moulinec,1998] H. Moulinec, P. Suquet, Comput. Methods Appl. Mech. Eng. 157 69-94 (1998).

[Murdoch-Bedeaux,1994] A.I. Murdoch, D. Bedeaux, Proc. R. Soc. Lond. A 445, 157-179 (1994).

[Murdoch,2007] A.I. Murdoch, J. Elasticity, 88, 113-140 (2007). 
[Nabarro,1947] F.R.N. Nabarro, Proc. Phys. Soc. 59, 256 (1947).

[Nielsen-Martin,1985] O.H. Nielsen and R.M. Martin, Phys. Rev. B 32, 37803791 (1985).

[Noll,1955] W. Noll, J. Ration. Mech. Anal. 4, 627-646 (1955).

[Nye,1953] J.F. Nye, Acta Metall., 1, 153 (1953).

[Pauli,1951] W. Pauli, in Handbuch der Physik, Band XXIV, Teil (Springer, Berlin, 1933), 83-272; Vol. V, Part 1 (1958).

[Peierls,1940] R. Peierls, Proc. Phys. Soc. 52, 34 (1940).

[Po,2014] G. Po, M. Lazar, D. Seif, N. Ghoniem, J. Mech. Phys. Solids 68, 161-178 (2014).

[Polizzotto,2001] C. Polizzotto, Int. J. Solids Structures 38, 7359-7380 (2001).

[Priester,2001] L. Priester, Mat. Sci. Eng. A309-310, 430-439 (2001).

[Schrödinger,1927] E. Schrödinger, Ann. Phys. (Leipzig) 82, 265 (1927).

[Schoeck,2002] G. Schoeck, Mater. Sci. Eng. A 333, 390-396 (2002).

[Seif,2014] D. Seif, G. Po, M. Mrovec, M. Lazar, C. Elsässer, P. Gumbsch, Phys. Rev. B 91, 184102 (2015).

[Shen,2004] C. Shen, Y. Wang, Y., Acta Mater. 52, 683 (2004). 
[Spearot,2007] D.E. Spearot, M.A. Tschopp, K.I. Jacob, D.L. McDowell, Acta Materialia 55, 705-714 (2007).

[Spearot,2008] D.E. Spearot, L. Capolungo, J. Qu, M. Cherkaoui, Computational Materials Science 42, 57-67 (2008).

[Sun,2016] X.-Y. Sun, V. Taupin, C. Fressengeas, P. Cordier, Int. J. Plast. 77, 75 (2016).

[Taupin,2013] V. Taupin, L. Capolungo, C. Fressengeas, A. Das, M. Upadhyay, J. Mech. Phys. Solids 61, 370 (2013).

[Taupin,2014] V. Taupin, L. Capolungo, C. Fressengeas, Int. J. Plast. 53, 179-192 (2014).

[Trautt,2012] Z.T. Trautt, Y. Mishin, Acta Mater. 60, 2407-2424 (2012).

[Tsai,1979] D.H. Tsai, J. Chem. Phys. 70, 1375-1382 (1979).

[Tschopp,2008] M.A. Tschopp, D.E. Spearot, D.L. McDowell, in: Hirth, J.P. (Ed.), Dislocations in Solids 14, 43 (2008).

[Tucker,2010] G.J. Tucker, J.A. Zimmerman, D.L. McDowell, Model. Simul. Mater. Sci. Eng. 18, 015002 (2010).

[Tucker,2012] G.J. Tucker, S. Tiwari, J.A. Zimmerman, D.L. McDowell, J. Mech. Phys. Solids 60, 471-486 (2012).

[Tucker,2015] G.J. Tucker, S.M. Foiles, Int. J. Plast. 65, 191-205 (2015). 
[Upadhyay,2013] M. Upadhyay, L. Capolungo, V. Taupin and C. Fressengeas, Phil. Mag. 93, 794 (2013).

[Zimmerman,2009] J.A. Zimmerman, D.J. Bammann, H. Gao, Int. J. Solids Struct. 46, 238-253 (2009). 